\title{
SINGULAR INTEGRAL OPERATORS ON A MANIFOLD WITH A DISTINGUISHED SUBMANIFOLD
}

\author{
YU.A. KORDYUKOV, V.A. PAVLENKO
}

\begin{abstract}
Let $X$ be a compact manifold without boundary and $X^{0}$ its smooth submanifold of codimension one. In this work we introduce classes of integral operators on $X$ with kernels $K_{A}(x, y)$, being smooth functions for $x \notin X^{0}$ and $y \notin X^{0}$, and admitting an asymptotic expansion of certain type, if $x$ or $y$ approaches $X^{0}$. For operators of these classes we prove theorems about action in spaces of conormal functions and composition. We show that the trace functional can be extended to a regularized trace functional $r$ - $\mathrm{rr}$ defined on some algebra $\mathcal{K}\left(X, X^{0}\right)$ of singular integral operators described above. We prove a formula for the regularized trace of the commutator of operators from this class in terms of associated operators on $X^{0}$. The proofs are based on theorems about pull-back and pushforward of conormal functions under maps of manifolds with distinguished codimension one submanifolds.
\end{abstract}

Ключевые слова: manifolds, singular integral operators, conormal functions, regularized trace, pull-back, push-forward

Mathematics Subject Classification: 47G10, 58J40, 47C05

\section{INTRODUCTION}

This paper is devoted to constructing and investigating of some classes of singular integral operators on a closed smooth manifold $X$ with a distinguished smooth codimension one submanifold $X^{0}$. A specific feature of the operators in these classes is that their kernels, $K_{A}(x, y)$, are smooth functions for $x \notin X^{0}$ and $y \notin X^{0}$ admitting an asymptotic expansion of a certain type as $x$ or $y$ approaches $X^{0}$.

First of all, we prove theorems on action in spaces of conormal functions and theorems on compositions for the operators in these classes. Then we construct an algebra $\mathcal{K}\left(X, X^{0}\right)$ of singular integral operators of this kind and a regularized trace functional $r$-Tr on it, which coincides with the trace functional on the operators with smooth kernel. Though the constructed functional does not have the trace property, we prove a formula for the regularized trace $\mathrm{r}-\operatorname{Tr}[A, B]$ of the commutator of operators $A$ and $B$ belonging to $\mathcal{K}\left(X, X^{0}\right)$ in terms of certain integral operators with smooth kernel on $X^{0}$ associated with $A$ and $B$.

One of the main motivations for our constructions is the desire to generalize the Lefschetz formula for a flow on a compact manifold preserving a codimension one foliation. In the case when the flow has no fixed points and its orbits are transversal to the leaves of the foliations, such a formula was proved in [1. The essential role in [1] is played by the following analytic result.

Let $M$ be a closed manifold and $\mathcal{F}$ be a smooth codimension one foliation on $M$. Suppose that $X_{t}: M \rightarrow M, t \in \mathbb{R}$ is a flow on $M$ which maps each leaf of $\mathcal{F}$ into a (possibly another) leaf.

Yu.A. Kordyukov, V.A. Pavlenko, Singular integral operators on A Manifold With A DISTINGUISHED SUBMANIFOLD.

(c) Kordyukov Yu.A., Pavlenko V.A. 2014.

The work is supported by RFBR (grant 12-01-00519-a).

Submitted March 13, 2014. 
Let $K$ be a leafwise smoothing operator on $M$, that is, an operator in the space $C^{\infty}(M)$ given by a family of integral operators with smooth kernel acting along the leaves of the foliation.

For each $f \in C_{0}^{\infty}(\mathbb{R})$, we define an operator $A_{f}$ in the space $C^{\infty}(M)$ by the formula

$$
A_{f}=\int_{\mathbb{R}} X_{t}^{*} \cdot f(t) d t \circ K
$$

where $X_{t}^{*}$ is the operator in $C^{\infty}(M)$ induced by the action of flow $X_{t}, X_{t}^{*} f(x)=f\left(X_{t}(x)\right)$. It is shown in [1] that, if the orbits of flow $X_{t}$ are transversal to the leaves, then, for each function $f \in C_{0}^{\infty}(\mathbb{R})$, operator $A_{f}$ is a trace class operator in the Hilbert space $L^{2}(M)$. Moreover, the functional $f \mapsto \operatorname{tr} A_{f}$ defines a distribution on $\mathbb{R}$. The use of distributions of such type allows us to define the Lefschetz number of flow $X_{t}$ as a distribution on $\mathbb{R}$.

In the case when flow $X_{t}$ has finitely many non-degenerate fixed points, belonging to compact leaves $\left\{L_{i}\right\}$, and the orbits of flow $X_{t}$ are transversal to all leaves except $\left\{L_{i}\right\}$, operator $A_{f}$ is not, generally speaking, a trace class operator. One can show that in this case operator $A_{f}$ belongs to the algebra $\mathcal{K}\left(M, M^{0}\right)$, where $M^{0}=\cup L_{i}$, and, therefore, its regularized trace $\mathrm{r}-\operatorname{Tr}\left(A_{f}\right)$ is well-defined. This fact allows us to define the Lefschetz number of flow $X_{t}$ in the case under consideration. These results are a part of our joint project with J. Alvarez Lopez and E. Leichtnam and will be discussed in subsequent papers.

Operator algebras associated with a compact manifold with a distinguished submanifold have been earlier constructed in papers of B.Yu. Sternin, V.E. Shatalov and A.Yu. Savin in connection with the study of boundary value problems for elliptic equations on a compact manifold, where the boundary conditions are given both on the boundary of the manifold and on smooth submanifolds (of codimension $\geqslant 1$ ) not being the boundary. Problems of such kind were considered for the first time by Sobolev [2]. A general setting of such problems and their study were given in [3] and, following this work, they are often called Sobolev problems. The operator algebra corresponding to Sobolev problems was constructed in [4]. It is obtained as an extension of the algebra of pseudodifferential operators by means of a special class of operators associated with the submanifold which are Green operators. It was shown in [5] that the theory of Sobolev problems can be represented as a relative theory, i.e., it is associated with the smooth embedding $i: X \hookrightarrow M$ of closed manifold. Relative theories are simpler and more elegant than theories which do not have this property. For instance, the computation of the index in a relative theory reduces to the computation of the index on smooth closed manifolds $M$ and $X$. On the contrary, in the theory of classical boundary value problems which is not relative (since it is associated with a manifold with boundary) the computation of the index is rather cumbersome. In [6, 7, 8, B.Yu. Sternin generalized the relative elliptic theory to the case when the submanifold is a stratified one presented as a union of transversally intersecting smooth submanifolds (see also [9, 10]).

The theory constructed in this paper is also a relative theory in the sense of B.Yu. Sternin [5]. To construct it, we make use of the methods of papers by Melrose [11, 12, 13], in particular, the geometric approach to constructing and studying algebras of singular integral operators suggested in these papers. The classes of operators and the notion of regularized trace introduced by us are analogues of the corresponding objects introduced earlier by Melrose for manifolds with corners.

The outline of the paper is as follows. In Section 2 we give the definition of conormal functions and conormal densities on a manifold $Z$ with a distinguished submanifold $Z^{0}$ and describe their basic properties. Submanifold $Z^{0}$ is not necessarily smooth, but it is represented as a union of smooth connected submanifolds of codimension 1 intersecting transversally. Such submanifolds will be called stratified. One of the main examples for us is as follows: $Z=X \times X, Z^{0}=$ $\left(X^{0} \times X\right) \cup\left(X \times X^{0}\right)$, where $X$ is a smooth manifold and $X^{0}$ is its smooth codimension one submanifold. The notion of conormal function introduced by us is a generalization of the classical notion of conormal function on a smooth submanifold introduced by Hörmander. A 
similar notion was introduced by Melrose for manifolds with corners. In Section 3 we construct various classes of singular integral operators and formulate theorems about action in spaces of conormal functions and about composition for operators of these classes. The proofs of these theorems are given in Section 4. They use theorems about pull-back and push-forward for conormal functions under maps of manifolds with distinguished submanifolds and constructions of some auxiliary manifolds. In Section 5, we define the regularized trace functional and prove its basic properties, in particular, theorem about the regularized trace of the commutator. In Appendices $\mathrm{A}$ and $\mathrm{B}$, we give the proofs of pull-back and push-forward theorems.

The authors express their gratitude to the referee for useful remarks.

\section{CONORMAL DENSITIES AND THEIR PROPERTIES}

In this section, we introduce the class of conormal functions on an arbitrary manifold $X$ with a distinguished stratified codimension one submanifold $X^{0}$.

2.1. Stratified submanifolds. Let $X$ be a smooth manifold of dimension $n$. A subset $X^{0} \subset$ $X$ will be called a stratified (codimension one) submanifold of manifold $X$, if $X^{0}$ is represented as a union of finitely many smooth submanifolds $X_{1}, X_{2}, \ldots, X_{r}$ of dimension $n-1$ intersecting transversally. We shall assume that submanifolds $X_{1}, X_{2}, \ldots, X_{r}$ are connected and we shall call them components of stratified submanifold $X^{0}$.

Here the transversal intersection has the following meaning. Let $p \in X^{0}$. Suppose that $p$ belongs to exactly $\ell$ components of the submanifold $X^{0}, \ell \geqslant 1$. Then there exists a local coordinate system $\varkappa: U \subset X \rightarrow \mathbb{R}^{\ell} \times \mathbb{R}^{n-\ell}$ with coordinates $\left(x, x^{0}\right) \in \mathbb{R}^{\ell} \times \mathbb{R}^{n-\ell}$ defined in a neighborhood of $p$ such that the intersections of the components of $X^{0}$ containing $p$ with $U$ are given by the equations $x_{d}=0$ for each $d \in\{1, \ldots, \ell\}$. Each such coordinate system will be called adapted at $p$. Without loss of generality, we can assume that $\varkappa(U)=D_{1} \times D_{2}$, where $D_{1} \subset \mathbb{R}^{\ell}$ and $D_{2} \subset \mathbb{R}^{n-\ell}$ are some open subsets. To be specific we will often assume that $p \in X_{1} \cap \ldots \cap X_{\ell}$ and $p \notin X_{\ell+1} \cup \ldots \cup X_{r}$, and adapted at $p$ coordinated system is chosen such that for any $d \in\{1, \ldots, \ell\}$ the intersection $X_{d} \cap U$ is given by the equation $x_{d}=0$. We will always consider regular local coordinate systems, that is, coordinate systems $\varkappa: U \subset X \rightarrow \mathbb{R}^{n}$ such that there exists a coordinate system $\bar{\varkappa}: V \subset X \rightarrow \mathbb{R}^{n}$ defined in an open set $V$ such that $\bar{U} \subset V$.

2.2. Index sets and families. Denote by $\mathbb{Q}_{1}$ the set of rational numbers represented in the form $z=\frac{p}{q}$, where $p, q \in \mathbb{Z}$ are coprime and $q$ is odd and by $\mathbb{Z}_{+}$the set of non-positive integers.

Definition 1. An index set is a set $E \subset \mathbb{Q}_{1} \times \mathbb{Z}_{+}$satisfying the following conditions:

1. E is bounded from below, i.e., there exists $N_{1} \in \mathbb{Q}_{1}$ such that, for each $(z, p) \in E$, we have $z \geqslant N_{1}$

2. $(z, p) \in E, p \geqslant q \Rightarrow(z, q) \in E$;

3. for each $N_{2} \in \mathbb{Q}_{1}$, the set $E \bigcap\left\{(z, p): z \leqslant N_{2}\right\}$ is finite;

4. $(z, p) \in E, j \in \mathbb{N} \Rightarrow(z+j, p) \in E$.

Definition 2. An index family $\mathcal{E}$ is said to be defined on a stratified submanifold $X^{0}=$ $X_{1} \cup \ldots \cup X_{r}$ if an index set $\mathcal{E}\left(X_{j}\right)=E_{j}, j=1, \ldots, r$ is assigned to each of its components $X_{j}$.

2.3. Conormal functions and their properties. Let $X$ be a smooth manifold and $X^{0}=$ $X_{1} \cup \ldots \cup X_{r}$ its stratified submanifold. Let $\mathcal{E}=\left(E_{1}, \ldots, E_{r}\right)$ be some index family on $X^{0}$. The definition of a conormal function at $p_{0} \in X^{0}$ will be given by induction by the number $\ell$ of components of $X^{0}$ containing $p_{0}$.

Basis of induction: $\ell=1$. Suppose that $p_{0}$ belongs to exactly one component, to be specific $p_{0} \in X_{1}, p_{0} \notin X_{2} \cup \ldots \cup X_{r}$. Take an adapted at $p_{0}$ coordinate $\varkappa: U \subset X \rightarrow \varkappa(U)=$ $D_{1} \times D_{2} \subset \mathbb{R} \times \mathbb{R}^{n-1}$. 
Definition 3. A function $u$ is said to be conormal at $p_{0}$ with respect to an index family $\mathcal{E}$ if there exists a neighborhood $V \subset U$ of $p_{0}, \varkappa(V)=(-\varepsilon, \varepsilon) \times V_{2}$, where $V_{2} \subset \mathbb{R}^{n-1}$, such that $u$ is defined and smooth on $V \backslash X^{0}$, and

$$
u \sim \sum_{(z, q) \in E_{1}} a_{z, q}\left(x^{0}\right) x^{z} \ln ^{q}|x|,
$$

where $a_{z, q} \in C^{\infty}\left(V_{2}\right)$. Here the symbol $\sim$ means that, for each $\alpha \in \mathbb{Z}_{+}, \beta \in \mathbb{Z}_{+}^{n-1}$ and $N \in \mathbb{N}$, there exists a constant $C=C_{\alpha \beta N}$ such that:

$$
\left|\left(x \partial_{x}\right)^{\alpha} \partial_{x^{0}}^{\beta}\left(u\left(x, x^{0}\right)-\sum_{\substack{(z, q) \in E_{1} \\ z \leqslant N}} a_{z, q}\left(x^{0}\right) x^{z} \ln ^{q}|x|\right)\right|<C|x|^{N+1}, \quad\left(x, x^{0}\right) \in(-\varepsilon, \varepsilon) \times V_{2}, x \neq 0 .
$$

Step of induction. Let $\ell \geqslant 2$. Suppose that the definition of conormal function at a point is given for each smooth manifold $Y$ with a distinguished stratified submanifold $Y^{0}$ on which an index family $\mathcal{E}^{0}$ is introduced and for each point $p_{1} \in Y^{0}$ under assumption that $p_{1}$ belongs to exactly $k$ components of $Y^{0}$ with $k<\ell$.

Suppose that $X$ is a smooth manifold with a distinguished stratified submanifold $X^{0}$, and $p_{0} \in X^{0}$, moreover, $p_{0}$ belongs to exactly $\ell$ components of $X^{0}$. To be specific we shall assume that $p_{0} \in X_{1} \cap \ldots \cap X_{\ell}$ and $p_{0} \notin X_{\ell+1} \cup \ldots \cup X_{r}$. We introduce an adapted at $p_{0}$ coordinate system $\varkappa: U \subset X \rightarrow \varkappa(U)=D_{1} \times D_{2} \subset \mathbb{R}^{\ell} \times \mathbb{R}^{n-\ell}$ such that $X_{j}$ is given by the equation $x_{j}=0$.

Consider the manifold $Z=\mathbb{R}^{\ell-1} \times \mathbb{R}^{n-\ell}$ with coordinates $\left(x_{2}, \ldots, x_{\ell}, x^{0}\right)$, where $x_{j} \in \mathbb{R}$, $j=2, \ldots, \ell, x^{0} \in \mathbb{R}^{n-\ell}$, equipped with the stratified submanifold $Z^{0}=\left\{x_{2}=0\right\} \cup \ldots \cup\left\{x_{\ell}=0\right\}$. We define an index set $\mathcal{E}^{\prime}$ on $Z^{0}$ by $\mathcal{E}^{\prime}\left(\left\{x_{j}=0\right\}\right)=E_{j}$, where $j=2, \ldots, \ell$. $Z^{0}$ consists of exactly $(\ell-1)$ components. Therefore, the notion of conormal function at an arbitrary point of $Z^{0}$ is well-defined by the induction hypothesis.

Definition 4. A function $u$ is said to be conormal at $p_{0}$ with respect to an index family $\mathcal{E}$ if there exists a neighborhood $V$ of $p_{0}, \varkappa(V)=(-\varepsilon, \varepsilon)^{\ell} \times V_{2}$, where $V_{2} \subset \mathbb{R}^{n-\ell}$ such that $u$ is defined and smooth on $V \backslash X^{0}$, and

$$
u \sim \sum_{(z, q) \in E_{1}} a_{z, q}\left(x_{2}, \ldots, x_{\ell}, x^{0}\right) x_{1}^{z} \ln ^{q}\left|x_{1}\right|,
$$

where the functions $a_{z, q}$ are conormal functions on $(-\varepsilon, \varepsilon)^{\ell-1} \times V_{2} \subset Z$ with respect to the index family $\mathcal{E}^{\prime}$.

The symbol $\sim$ means that there are $M_{2}, \ldots, M_{\ell} \in \mathbb{R}$ such that for each $\alpha \in \mathbb{Z}_{+}^{\ell}$ and $\beta \in \mathbb{Z}_{+}^{n-\ell}$ and for each $N \in \mathbb{N}$ there exists a constant $C=C_{\alpha \beta N}$ such that:

$$
\begin{aligned}
& \left|\left(x \partial_{x}\right)^{\alpha} \partial_{x^{0}}^{\beta}\left(u\left(x_{1}, x_{2}, \ldots, x_{\ell}, x^{0}\right)-\sum_{\substack{(z, q) \in E_{1} \\
z \leqslant N_{1}}} a_{z, q}\left(x_{2}, \ldots, x_{\ell}, x^{0}\right) x_{1}^{z} \ln ^{q}\left|x_{1}\right|\right)\right| \\
& <C\left|x_{2}\right|^{M_{2}} \cdot \ldots \cdot\left|x_{\ell}\right|^{M_{\ell}}\left|x_{1}\right|^{N+1}, \quad\left(x, x^{0}\right) \in(-\varepsilon, \varepsilon)^{\ell} \times V_{2}, x_{j} \neq 0 .
\end{aligned}
$$

One can show that the definition of a conormal function at a point is independent of the choice of local coordinate system. In particular, the expansion of type (1) holds for each of variables $x_{2}, \ldots, x_{\ell}$.

Definition 5. A function $u$ is said to be a conormal function on a manifold $X$ with a stratified submanifold $X^{0}$ with respect to an index family $\mathcal{E}$, if it is smooth on $X \backslash X^{0}$ and conormal at each point $p_{0} \in X^{0}$ with respect to $\mathcal{E}$.

The class of conormal functions on a manifold $X$ with a distinguished submanifold $X^{0}$ with respect to an index family $\mathcal{E}$ will be denoted by $\mathcal{A}_{p h g}^{\mathcal{E}}\left(X, X^{0}\right)$. 
Remark 1. (1) If $\mathcal{E}$ is the trivial index family, i.e. $\mathcal{E}\left(X_{j}\right)=\left\{(\ell, 0): \ell \in \mathbb{Z}_{+}\right\}$for each $j=1, \ldots, r$, then $\mathcal{A}_{\text {phg }}^{\mathcal{E}}\left(X, X^{0}\right)=C^{\infty}(X)$.

(2) For each function $u \in \mathcal{A}_{\text {phg }}^{\mathcal{E}_{1}}\left(X, X^{0}\right)$ and for each function $v \in \mathcal{A}_{\text {phg }}^{\mathcal{E}_{2}}\left(X, X^{0}\right)$ the inclusions $u+v \in \mathcal{A}_{\text {phg }}^{\mathcal{E}_{1} \cup \mathcal{E}_{2}}\left(X, X^{0}\right)$ hold true as well as $u v \in \mathcal{A}_{\text {phg }}^{\mathcal{E}_{1}+\mathcal{E}_{2}}\left(X, X^{0}\right)$.

Example 1. In the simplest example $X=\mathbb{R}^{2}$ and $X^{0}=(\mathbb{R} \times\{0\}) \cup(\{0\} \times \mathbb{R})$ the function $u(x, y)=\sqrt{x^{2}+y^{2}}$ on $X$ is not conormal at $(0,0)$.

The notion of conormality is easily generalized to sections of a vector bundle.

Definition 6. Let $X$ be a smooth manifold, $X^{0}$ be a stratified submanifold, $G$ be a smooth vector bundle on $X$. A section $\mu$ is said to be a conormal section, $\mu \in \mathcal{A}_{\text {phg }}^{\mathcal{E}}\left(X, X^{0}, G\right)$, if in each trivialization $\left.G\right|_{U} \cong U \times \mathbb{C}^{r}$ of $G$ over the coordinate neighborhood $U \subset X$ the section $\mu$ reads as $\mu(x)=\left(x,\left(u_{1}(x), \ldots, u_{r}(x)\right), x \in U\right.$, where $u_{j} \in \mathcal{A}_{\text {phg }}^{\mathcal{E}}\left(X, X^{0}\right), j=1, \ldots, r$.

2.4. Conormal densities. We shall consider operators acting on half-densities. We recall that a smooth $s$-density $\mu$ on a smooth manifold $M$ of dimension $n$ is written in an arbitrary local coordinate system as $\mu=u\left(x_{1}, \ldots, x_{n}\right)\left|d x_{1} \ldots d x_{n}\right|^{s}$, where $u$ is a smooth function. Smooth $s$ densities are smooth sections of a certain line bundle $\Omega_{M}^{s}$ on $M$. We shall denote by $C^{\infty}\left(M, \Omega_{M}^{s}\right)$ the space of smooth $s$-densities on $M$.

Definition 7. Let $X$ be a smooth manifold and $X^{0}=X_{1} \cup \ldots \cup X_{r}$ be its stratified submanifold. An s-density $\mu$ on $X$ is said to be conormal with respect to an index family $\mathcal{E}$ if in each adapted local coordinate system with coordinates $\left(x, x^{0}\right) \in \mathbb{R}^{\ell} \times \mathbb{R}^{n-\ell}$ it reads as

$$
\mu=\frac{u\left(x, x^{0}\right)}{|x|^{s}}\left|d x d x^{0}\right|^{s}=u\left(x, x^{0}\right)\left|\frac{d x}{x} d x^{0}\right|^{s},
$$

where $u$ is a conormal function with respect to $\mathcal{E}$.

The space of conormal $s$-densities on $X$ with respect to an index family $\mathcal{E}$ is naturally isomorphic to the space $\mathcal{A}_{p h g}^{\mathcal{E}}\left(X, X^{0}, \Omega_{X, X_{0}}^{s}\right)$ of conormal sections of a certain line bundle $\Omega_{X, X_{0}}^{s}$ on $X$. The construction of $\Omega_{X, X_{0}}^{s}$ is similar to the construction of the bundle of $b$-densities on a manifold with corners suggested by Melrose and will be omitted.

\section{Singular integral operators}

In this section, we introduce classes of singular integral operators on a manifold with a distinguished submanifold.

3.1. Classes $\mathcal{K}^{\mathcal{E}_{1}, \mathcal{E}_{2}}\left(X, X^{0} ; Y, Y^{0}\right)$. Let $X$ and $Y$ be compact smooth manifolds, $\operatorname{dim} X=n$, $\operatorname{dim} Y=m, X^{0}, Y^{0}$ be smooth codimension 1 submanifolds of $X$ and $Y$, respectively.

A half-density $k_{A} \in C^{\infty}\left((X \times Y) \backslash\left(\left\{X^{0} \times Y\right\} \cup\left\{X \times Y^{0}\right\}\right), \Omega_{X \times Y}^{\frac{1}{2}}\right)$ defines an operator

$$
A: C_{0}^{\infty}\left(Y \backslash Y^{0}, \Omega_{Y}^{\frac{1}{2}}\right) \rightarrow C^{\infty}\left(X \backslash X^{0}, \Omega_{X}^{\frac{1}{2}}\right),
$$

whose action on a half-density $\mu \in C_{0}^{\infty}\left(Y \backslash Y^{0}, \Omega_{Y}^{\frac{1}{2}}\right)$ is given by the formula

$$
A \mu=\int_{Y} k_{A} \mu .
$$

Half-density $k_{A}$ is called the kernel of the operator $A$.

Let us explain the meaning of the expression in the right-hand side of (2). Kernel $k_{A}$ and half-density $\mu$ can be written as

$$
k_{A}=K_{A}\left(p_{1}, p_{2}\right)\left|d v_{X}\left(p_{1}\right) d v_{Y}\left(p_{2}\right)^{\mid \frac{1}{2}}, \quad \mu=u\left(p_{2}\right)\right| d v_{Y}\left(p_{2}\right)^{\frac{1}{2}},
$$


where $K_{A} \in C^{\infty}\left((X \times Y) \backslash\left(\left\{X^{0} \times Y\right\} \cup\left\{X \times Y^{0}\right\}\right)\right), u \in C_{0}^{\infty}\left(Y \backslash Y^{0}\right),\left|d v_{X}\right|$ is a positive smooth density on $X$ and $\left|d v_{Y}\right|$ is a positive smooth density on $Y$. Then their product

$$
k_{A} \mu=K_{A}\left(p_{1}, p_{2}\right) u\left(p_{2}\right)\left|d v_{X}\left(p_{1}\right)\right|^{\frac{1}{2}}\left|d v_{Y}\left(p_{2}\right)\right|
$$

is a density on $Y$. It can be integrated over $Y$ resulting in a half-density on $X$ :

$$
\int_{Y} k_{A} \mu=\left(\int_{Y} K_{A}\left(p_{1}, p_{2}\right) u\left(p_{2}\right)\left|d v_{Y}\left(p_{2}\right)\right|\right)\left|d v_{X}\left(p_{1}\right)\right|^{\frac{1}{2}} .
$$

It is easy to see that formula (2) agrees with the standard expression for the integral operator with kernel $K_{A}$ :

$$
A \mu=A u\left(p_{1}\right)\left|d v_{X}\left(p_{1}\right)\right|^{\frac{1}{2}}, \quad A u\left(p_{1}\right)=\int_{Y} K_{A}\left(p_{1}, p_{2}\right) u\left(p_{2}\right) d v_{Y}\left(p_{2}\right) .
$$

If $p_{1} \notin X^{0}$, the integral in the right-hand side converges.

Consider the stratified submanifold $\left\{X^{0} \times Y\right\} \cup\left\{X \times Y^{0}\right\}$ of the manifold $X \times Y$. Each index family $\mathcal{E}$ on $\left\{X^{0} \times Y\right\} \cup\left\{X \times Y^{0}\right\}$ is written as $\mathcal{E}=\left(\mathcal{E}_{1}, \mathcal{E}_{2}\right)$, where $\mathcal{E}_{1}$ is an index family on $X^{0} \times Y$ and $\mathcal{E}_{2}$ an index family on $X \times Y^{0}$. In what follows, we shall also consider index family $\mathcal{E}_{1}$ as an index family on $X^{0}$ and $\mathcal{E}_{2}$ as an index family on $Y^{0}$.

Definition 8. Let $\mathcal{E}_{1}$ be an index family on $X^{0}, \mathcal{E}_{2}$ be an index family on $Y^{0}$ and $\left(\mathcal{E}_{1}, \mathcal{E}_{2}\right)$ the corresponding index family on $\left\{X^{0} \times Y\right\} \cup\left\{X \times Y^{0}\right\}$. We shall say that an integral operator $A$ given by (2) belongs to the class $\mathcal{K}^{\mathcal{E}_{1}, \mathcal{E}_{2}}\left(X, X^{0} ; Y, Y^{0}\right)$ if

$$
k_{A} \in \mathcal{A}_{p h g}^{\left(\mathcal{E}_{1}, \mathcal{E}_{2}\right)}\left(X \times Y,\left\{X^{0} \times Y\right\} \cup\left\{X \times Y^{0}\right\}, \Omega_{X \times Y,\left\{X^{0} \times Y\right\} \cup\left\{X \times Y^{0}\right\}}^{\frac{1}{2}}\right) .
$$

It is clear that $\mathcal{K}^{\mathcal{E}_{1}, \mathcal{E}_{2}}\left(X, X^{0} ; Y, Y^{0}\right)$ is a linear space.

Example 2. In the simplest example $X=Y=\mathbb{R}$ and $X^{0}=Y^{0}=\{0\}$, integral operator $A$ with kernel

$$
k_{A}=C x^{\alpha} y^{\beta} \ln ^{p}|x| \ln ^{q}|y|\left|\frac{d x}{x} \frac{d y}{y}\right|^{1 / 2}, \quad x, y \in \mathbb{R} \backslash\{0\},
$$

belongs to the class $\mathcal{K}^{\mathcal{E}_{1}, \mathcal{E}_{2}}\left(X, X^{0} ; Y, Y^{0}\right)$ with $\mathcal{E}_{1}\left(X^{0}\right)=\left\{(\alpha+j, k): j \in \mathbb{Z}_{+}, k=0,1, \ldots, p\right\}$, $\mathcal{E}_{2}\left(Y^{0}\right)=\left\{(\beta+j, k): j \in \mathbb{Z}_{+}, k=0,1, \ldots, q\right\}$.

For an index set $E$, we let $\inf E:=\inf \{z:(z, p) \in E\}$. If $\mathcal{E}$ is an index family on a stratified submanifold $X^{0}=X_{1} \cup \ldots \cup X_{r}$ of manifold $X$, we denote $\inf \mathcal{E}=\inf _{j=1, \ldots, r} \inf \mathcal{E}\left(X_{j}\right)$.

Theorem 1. Let $A \in \mathcal{K}^{\mathcal{E}_{1}, \mathcal{E}_{2}}\left(X, X^{0} ; Y, Y^{0}\right)$. Then, for each index family $\mathcal{F}$ on $Y^{0}$, satisfying the condition $\inf \left(\mathcal{E}_{2}+\mathcal{F}\right)>0$, operator $A$ can be extended to the operator

$$
A: \mathcal{A}_{p h g}^{\mathcal{F}}\left(Y, Y^{0}, \Omega_{Y, Y^{0}}^{\frac{1}{2}}\right) \rightarrow \mathcal{A}_{p h g}^{\mathcal{E}_{1}}\left(X, X^{0}, \Omega_{X, X^{0}}^{\frac{1}{2}}\right) .
$$

Theorem 2. If $A \in \mathcal{K}^{\mathcal{E}_{1}, \mathcal{E}_{2}}\left(X, X^{0} ; Y, Y^{0}\right)$ and $B \in \mathcal{K}^{\mathcal{F}_{2}, \mathcal{F}_{3}}\left(Y, Y^{0} ; Z, Z^{0}\right)$, then under condition $\inf \left(\mathcal{E}_{2}+\mathcal{F}_{2}\right)>0$ their composition $C=A \circ B$ is well-defined and belongs to the class $\mathcal{K}^{\mathcal{E}_{1}, \mathcal{F}_{3}}\left(X, X^{0} ; Z, Z^{0}\right)$.

3.2. Normal coordinates near a submanifold. Let $M$ be a compact manifold, $M^{0}$ be its smooth submanifold. We choose a Riemannian metric $g_{M}$ on $M$ and consider the normal bundle $N\left(M^{0}\right):=T M / T M^{0} \cong\left(T M^{0}\right)^{\perp}$. We recall that the exponential map exp : $N\left(M^{0}\right) \rightarrow M$ of Riemannian metric $g_{M}$ for submanifold $M^{0}$ is defined as follows. Let $v \in N_{x}\left(M^{0}\right), x \in M$. There exists the unique geodesic $\gamma:(-\infty,+\infty) \rightarrow M$, passing through $x$ with the velocity vector $v$, that is, such that $\gamma(0)=x, \dot{\gamma}(0)=v$. Then $\exp (v):=\gamma(1)$. 
One can identify $M^{0}$ with the zero section of bundle $N\left(M^{0}\right)$ that allows us to consider $M^{0}$ both as a submanifold of $M$ and as a submanifold of $N\left(M^{0}\right)$. The following proposition holds.

Proposition 1. There exists a neighborhood $U \supset M^{0}$ in $N\left(M^{0}\right)$ such that the restriction $\left.\exp \right|_{U}$ to $U$ is a diffeomorphism of $U$ on some neighborhood $\exp (U)$ of submanifold $M^{0}$.

The set $\exp (U)$ is called a tubular neighborhood of $M^{0}$ in $M$. Without loss of generality, we can assume that $\exp (U)$ is an $\varepsilon$-neighborhood of $M^{0}$ for some $\varepsilon>0$.

We suppose that submanifold $M^{0}$ is of codimension one, and normal bundle $N\left(M^{0}\right)$ is trivial. Assume that $U \supset M^{0}$ is as in Proposition 1 and choose $p \in \exp U$. This point is in a one-to-one correspondence with a pair $\left(x, x^{0}\right) \in N\left(M^{0}\right)$, where $x^{0} \in M^{0}$ and $x \in N_{x^{0}}\left(M^{0}\right)$, $\exp (x)=p$. Since the Riemannian metric determines an isomorphism $N_{x^{0}}\left(M^{0}\right) \cong \mathbb{R}$, one can consider $x \in \mathbb{R}$. Thus, each point $p$ in the tubular neighborhood $\exp (U)$ is uniquely determined by a pair $\left(x, x^{0}\right)$, where $x \in \mathbb{R}$ and $x^{0} \in M^{0}$. The map $\exp (U) \rightarrow(-\varepsilon, \varepsilon) \times M^{0}, p \mapsto\left(x, x^{0}\right)$ will be called a normal coordinate system near $M^{0}$.

3.3. Classes $\mathcal{K}^{\mathcal{E}_{1}, \mathcal{E}_{2}, \mathcal{E}_{O}}$. Let $X$ be a compact smooth manifold of dimension $n, g_{X}$ be a Riemannian metric on $X, X^{0}=X_{1} \cup \ldots \cup X_{r}$ be its smooth codimension one submanifold. Thus, submanifolds $X_{1}, \ldots, X_{r}$ are mutually disjoint. Suppose that the normal bundles of $X_{1}, \ldots, X_{r}$ are trivial.

Consider an operator $A: C_{0}^{\infty}\left(X \backslash X^{0}, \Omega_{X}^{\frac{1}{2}}\right) \rightarrow C^{\infty}\left(X \backslash X^{0}, \Omega_{X}^{\frac{1}{2}}\right)$ with a kernel

$$
k_{A} \in C^{\infty}\left((X \times X) \backslash\left(\left\{X^{0} \times X\right\} \cup\left\{X \times X^{0}\right\}\right), \Omega_{X \times X}^{\frac{1}{2}}\right) .
$$

Hereafter $\left|d x^{0}\right|$ is a fixed positive smooth density on $X^{0}$.

We choose a normal coordinate system with coordinates $\left(x, x^{0}\right) \in(-\varepsilon, \varepsilon) \times X^{0}$ in a tubular neighborhood $\exp (U)=V$ of $X^{0}$. Let $\left(x_{1}, x_{2}, x_{1}^{0}, x_{2}^{0}\right)$ be the corresponding coordinates on $V \times V$. We denote $\Pi_{\varepsilon}=\left\{(x, s) \in \mathbb{R}^{2}: 0<|x|<\varepsilon,\left|\frac{x}{s}\right|<\varepsilon\right\}$ and introduce a coordinate system $\left(x, s, x_{1}^{0}, x_{2}^{0}\right) \in \Pi_{\varepsilon} \times X^{0} \times X^{0}$ on the set $\left(V \backslash X^{0}\right) \times\left(V \backslash X^{0}\right)$ by the formulae

$$
x=x_{1}, \quad s=\frac{x_{1}}{x_{2}} .
$$

Then the half-density

$$
k_{A}=K_{A}\left(x_{1}, x_{2}, x_{1}^{0}, x_{2}^{0}\right)\left|\frac{d x_{1}}{x_{1}} \frac{d x_{2}}{x_{2}} d x_{1}^{0} d x_{2}^{0}\right|^{\frac{1}{2}}
$$

in the local coordinate system $\left(x, s, x_{1}^{0}, x_{2}^{0}\right)$ is written as

$$
k_{A}=K_{A}\left(x, \frac{x}{s}, x_{1}^{0}, x_{2}^{0}\right)\left|\frac{d x}{x} \frac{d s}{s} d x_{1}^{0} d x_{2}^{0}\right|^{\frac{1}{2}} .
$$

We define a function $\widetilde{K}_{A}$ on $\Pi_{\varepsilon} \times X^{0} \times X^{0}$ by

$$
\widetilde{K}_{A}\left(x, s, x_{1}^{0}, x_{2}^{0}\right)=K_{A}\left(x, \frac{x}{s}, x_{1}^{0}, x_{2}^{0}\right) .
$$

Let $\mu \in C_{0}^{\infty}\left(X, \Omega_{X}^{\frac{1}{2}}\right)$, supp $\mu \subset V$. We write $\mu=u\left(x, x^{0}\right)\left|\frac{d x}{x} d x^{0}\right|^{\frac{1}{2}}$, where $u \in C_{0}^{\infty}(V) \cong$ $C_{0}^{\infty}\left((-\varepsilon, \varepsilon) \times X^{0}\right)$. Then

$$
\left.A \mu\right|_{V}=\left(\int_{X^{0}} \int_{-\infty}^{+\infty} \widetilde{K}_{A}\left(x, s, x_{1}^{0}, x_{2}^{0}\right) u\left(\frac{x}{s}, x_{2}^{0}\right) \frac{d s}{s} d x_{2}^{0}\right)\left|\frac{d x}{x} d x_{1}^{0}\right|^{\frac{1}{2}} .
$$

Definition 9. Let $\mathcal{E}_{1}, \mathcal{E}_{2}$ be index families on $X^{0}, \mathcal{E}_{O}=\left\{\mathcal{E}_{O, i j}: i, j=1, \ldots, r\right\}$, where $\mathcal{E}_{O, i j}$ is an index set for each $i, j=1, \ldots, r$. We say that an operator $A$ belongs to the class $\mathcal{K}^{\mathcal{E}_{1}, \mathcal{E}_{2}, \mathcal{E}_{O}}\left(X, X^{0}\right)$ if 
(1) Kernel $k_{A}$ is a conormal half-density on $(X \times X) \backslash\left(X^{0} \times X^{0}\right)$ with distinguished submanifold $\left\{X^{0} \times\left(X \backslash X^{0}\right)\right\} \cup\left\{\left(X \backslash X^{0}\right) \times X^{0}\right\}$ with respect to the index family $\widehat{E}_{1}=\left(\mathcal{E}_{1}, \mathcal{E}_{2}\right)$ :

$$
\widehat{E}_{1}\left(X_{i} \times\left(X \backslash X^{0}\right)\right)=\mathcal{E}_{1}\left(X_{i}\right), \quad \widehat{E}_{1}\left(\left(X \backslash X^{0}\right) \times X_{j}\right)=\mathcal{E}_{2}\left(X_{j}\right) .
$$

(2) Function $\widetilde{K}_{A}\left(x, s, x_{1}^{0}, x_{2}^{0}\right)$ on $\Pi_{\varepsilon} \times X^{0} \times X^{0}$ is conormal at the submanifold $\{0\} \times(\mathbb{R} \backslash$ $\{0\}) \times X^{0} \times X^{0}$ with respect to index family $\widehat{E}_{2}$ :

$$
\widehat{E}_{2}\left(\{0\} \times(\mathbb{R} \backslash\{0\}) \times X_{i} \times X_{j}\right)=\mathcal{E}_{O, i j} .
$$

(3) Function $\widehat{K}_{A}$ on $\left\{(x, \tau) \in \mathbb{R}^{2}:|x|<\varepsilon,|x \tau|<\varepsilon\right\} \times X^{0} \times X^{0}$ defined by

$$
\widehat{K}_{A}\left(x, \tau, x_{1}^{0}, x_{2}^{0}\right)=K_{A}\left(x, x \tau, x_{1}^{0}, x_{2}^{0}\right),
$$

is conormal at the submanifold $\left(\{0\} \times \mathbb{R} \times X^{0} \times X^{0}\right) \cup\left((-\varepsilon, \varepsilon) \times\{0\} \times X^{0} \times X^{0}\right)$ with respect to the index family $\widehat{E}_{3}=\left(\mathcal{E}_{O}, \mathcal{E}_{2}\right)$

$$
\widehat{E}_{3}\left(\{0\} \times \mathbb{R} \times X_{i} \times X_{j}\right)=\mathcal{E}_{O, i j}, \quad \widehat{E}_{3}\left((-\varepsilon, \varepsilon) \times\{0\} \times X_{i} \times X_{j}\right)=\mathcal{E}_{2}\left(X_{j}\right) .
$$

(4) Function $\widehat{\widetilde{K}}_{A}$ on $\left\{(t, x) \in \mathbb{R}^{2}:|t x|<\varepsilon,|x|<\varepsilon\right\} \times X^{0} \times X^{0}$ defined by

$$
\widehat{\widetilde{K}}_{A}\left(t, x, x_{1}^{0}, x_{2}^{0}\right)=K_{A}\left(t x, x, x_{1}^{0}, x_{2}^{0}\right),
$$

is conormal at the submanifold $\left(\{0\} \times(-\varepsilon, \varepsilon) \times X^{0} \times X^{0}\right) \cup\left(\mathbb{R} \times\{0\} \times X^{0} \times X^{0}\right)$ with respect to the index family $\widehat{E}_{4}=\left(\mathcal{E}_{1}, \mathcal{E}_{O}\right)$

$$
\widehat{E}_{4}\left(\{0\} \times(-\varepsilon, \varepsilon) \times X_{i} \times X_{j}\right)=\mathcal{E}_{1}\left(X_{i}\right), \quad \widehat{E}_{4}\left(\mathbb{R} \times\{0\} \times X_{i} \times X_{j}\right)=\mathcal{E}_{O, i j} .
$$

It is clear that the class $\mathcal{K}^{\mathcal{E}_{1}, \mathcal{E}_{2}, \mathcal{E}_{O}}\left(X, X^{0}\right)$ is a linear space.

Remark 2. One can show that $\mathcal{K}^{\mathcal{E}_{1}, \mathcal{E}_{2}}\left(X, X^{0}\right) \subset \mathcal{K}^{\mathcal{E}_{1}, \mathcal{E}_{2}, \mathcal{E}_{O}}\left(X, X^{0}\right)$, where $\mathcal{E}_{O, i j}=\mathcal{E}_{1}\left(X_{i}\right)+$ $\mathcal{E}_{2}\left(X_{j}\right)$.

Example 3. In the simplest example $X=\mathbb{R}$ and $X^{0}=\{0\}$ the integral operator $A$ with the kernel

$$
k_{A}=x^{\alpha} y^{\beta}\left(x^{2}+y^{2}\right)^{\frac{\gamma}{2}} \ln ^{p}|x| \ln ^{q}|y| \ln ^{r}\left(x^{2}+y^{2}\right)\left|\frac{d x}{x} \frac{d y}{y}\right|^{1 / 2},
$$

belongs to the class $\mathcal{K}^{\mathcal{E}_{1}, \mathcal{E}_{2}, \mathcal{E}_{O}}\left(X, X^{0}\right)$, where $\mathcal{E}_{1}\left(X^{0}\right)=\left\{(\alpha+j, k): j \in \mathbb{Z}_{+}, k=0,1, \ldots, p\right\}$, $\mathcal{E}_{2}\left(X^{0}\right)=\left\{(\beta+j, k): j \in \mathbb{Z}_{+}, k=0,1, \ldots, q\right\}$ and $\mathcal{E}_{O}=\left\{(\alpha+\beta+\gamma+j, k): j \in \mathbb{Z}_{+}, k=\right.$ $0,1, \ldots, p+q+r\}$.

Let $E_{1}, E_{2}$ be arbitrary index sets. We let

$$
E_{1} \cup E_{2}=E_{1} \cup E_{2} \cup\left\{\left(z, p_{1}+p_{2}+1\right):\left(z, p_{1}\right) \in E_{1},\left(z, p_{2}\right) \in E_{2}\right\} .
$$

Theorem 3. Let $A \in \mathcal{K}^{\mathcal{E}_{1}, \mathcal{E}_{2}, \mathcal{E}_{O}}\left(X, X^{0}\right)$. Then, for each index family $\mathcal{F}$ satisfying the condition $\inf \left(\mathcal{E}_{2}+\mathcal{F}\right)>0$, operator $A$ can be extended to the operator

$$
A: \mathcal{A}_{p h g}^{\mathcal{F}}\left(X, X^{0}, \Omega_{X, X^{0}}^{\frac{1}{2}}\right) \rightarrow \mathcal{A}_{p h g}^{\mathcal{G}}\left(X, X^{0}, \Omega_{X, X^{0}}^{\frac{1}{2}}\right),
$$

where

$$
\mathcal{G}\left(X_{i}\right)=\mathcal{E}_{1}\left(X_{i}\right) \bar{\bigcup}\left(\overline{\bigcup_{j}}\left(\mathcal{F}\left(X_{j}\right)+\mathcal{E}_{O, i j}\right)\right), \quad i=1, \ldots, r
$$




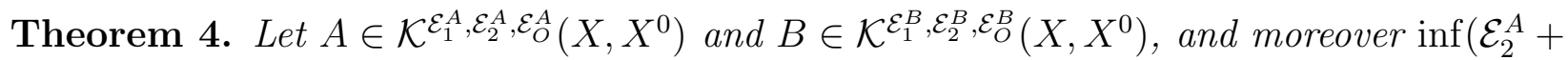
$\left.\mathcal{E}_{1}^{B}\right)>0$. Then the composition $C=A \circ B$ is well-defined and belongs to the class

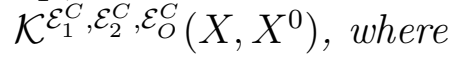

$$
\begin{gathered}
\mathcal{E}_{1}^{C}\left(X_{i}\right)=\mathcal{E}_{1}^{A}\left(X_{i}\right) \bar{\bigcup}\left(\bigcup_{k}\left(\mathcal{E}_{O, i k}^{A}+\mathcal{E}_{1}^{B}\left(X_{k}\right)\right)\right), \\
\mathcal{E}_{2}^{C}\left(X_{j}\right)=\mathcal{E}_{2}^{B}\left(X_{j}\right) \bar{\bigcup}\left(\bigcup_{k}\left(\mathcal{E}_{2}^{A}\left(X_{k}\right)+\mathcal{E}_{O, k j}^{B}\right)\right), \\
\mathcal{E}_{O, i j}^{C}=\left(\bigcup_{k}\left(\mathcal{E}_{O, i k}^{A}+\mathcal{E}_{O, k j}^{B}\right)\right) \bar{\bigcup}\left(\mathcal{E}_{1}^{A}\left(X_{i}\right)+\mathcal{E}_{2}^{B}\left(X_{j}\right)\right) .
\end{gathered}
$$

Remark 3. The results obtained in the paper are likely to be extended to the case when the normal bundle of $X^{0}$ is nontrivial. In order to do it, one needs to pass to the corresponding double covering and to work with $\mathbb{Z}_{2}$-invariant operators. An appropriate technique was developed for manifolds with corners in [14].

\section{PROOFS OF MAIN THEOREMS}

In this section we provide the proofs of Theorems 1, 2, 3 and 4. As it has been already said in Introduction, our approach to constructing and studying classes of singular integral operators is a generalization of the geometric approach suggested by Melrose ([11, 12, 13], see also [15]). A specific feature of Melrose's approach is that classes of operators are defined by means of certain conditions on a kernel $k_{A}$ of an operator $A$ in a given class. These conditions are either the conormality conditions for kernel $k_{A}$ or for some half-density $\hat{k}_{A}$ being the pull-back of kernel $k_{A}$ to an auxiliary manifold associated with $X \times X$. In order to relate operator $A$ with the kernel $\hat{k}_{A}$, the action of the integral operator $A$ on half-densities is expressed in terms of pull-back and push-forward operators. Thus, the study of the given class of integral operators is reduced to employing pull-back and push-forward operators and their properties. Therefore, we begin with a discussion of pull-back and push-forward operators.

4.1. Pull-backs. Let us recall the definitions of the pull-back operator associated with a map of smooth manifolds.

Let $X$ and $Y$ be smooth manifolds, $f: X \rightarrow Y$ a smooth map. For each vector bundle $p: G \rightarrow Y$ on $Y$, we define a vector bundle $p_{1}: f^{*} G \rightarrow X$ as follows:

$$
f^{*} G:=\left\{(x, v) \mid x \in X ; v \in G_{f(x)}\right\}, \quad p_{1}(x, v):=x .
$$

Definition 10. The pull-back operator is a linear operator

$$
f^{*}: C^{\infty}(Y, G) \rightarrow C^{\infty}\left(X, f^{*} G\right)
$$

given for each $s \in C^{\infty}(Y, G)$ by the identity

$$
f^{*} s(x)=(x, s(f(x))), \quad x \in X .
$$

Let $X$ and $Y$ be smooth manifolds of dimension $n$ and $m$ respectively, $X^{0}=X_{1} \cup \ldots \cup X_{r}$ and $Y^{0}=Y_{1} \cup \ldots \cup Y_{r^{0}}$ stratified submanifolds of $X$ and $Y$ respectively.

Definition 11. A smooth map $f: X \rightarrow Y$ is said to be relative if for each $p \in X^{0}$ the following condition holds. To be specific we suppose that $p \in X_{1} \cap \ldots \cap X_{\ell}, p \notin X_{\ell+1} \cup \ldots \cup X_{r}$ and $f(p) \in Y_{1} \cap \ldots \cap Y_{\ell_{0}}, f(p) \in Y_{\ell_{0}+1} \cup \ldots \cup Y_{r_{0}}$. We choose an adapted at $p$ coordinate system with coordinates $\left(x, x^{0}\right) \in \mathbb{R}^{\ell} \times \mathbb{R}^{n-\ell}$ defined in a neighborhood $U_{p}$, and an adapted at $f(p)$ coordinate system with coordinates $\left(y, y^{0}\right) \in \mathbb{R}^{\ell_{0}} \times \mathbb{R}^{m-\ell_{0}}$. In these coordinates map $f$ is written as

$$
y_{i}=f_{i}\left(x, x^{0}\right), \quad i=1, \ldots, \ell_{0} ; \quad y_{i}^{0}=f_{i}\left(x, x^{0}\right), \quad i=\ell_{0}+1, \ldots, m
$$


Then there exist smooth functions $a_{i}, i=1, \ldots, \ell_{0}$, such that $a_{i}\left(x, x^{0}\right) \neq 0$ and in some neighborhood of $p$ we have a representation:

$$
f_{i}\left(x_{1}, \ldots, x_{\ell}, x^{0}\right)=a_{i}\left(x, x^{0}\right) \prod_{j=1}^{\ell} x_{j}^{\gamma_{i j}},
$$

where $\gamma_{i j}$ are non-negative integers, $i=1, \ldots, \ell_{0}, j=1, \ldots, \ell$.

Numbers $\gamma_{i j}$ depend only on components $X_{j}$ and $Y_{i}$ and will be denoted by $e_{f}\left(X_{j}, Y_{i}\right)$. Observe that the definition of relative map implies that $f^{-1}\left(Y^{0}\right) \subset X^{0}$.

Theorem 5. Let $G$ be a line bundle on $Y, \mathcal{E}^{0}$ be an index family on a submanifold $Y^{0}$. Then for each relative map $f:\left(X, X^{0}\right) \rightarrow\left(Y, Y^{0}\right)$ operator $f^{*}$ can be extended to an operator

$$
f^{*}: \mathcal{A}_{p h g}^{\mathcal{E}^{0}}\left(Y, Y^{0}, G\right) \rightarrow \mathcal{A}_{p h g}^{\mathcal{E}}\left(X, X^{0}, f^{*} G\right),
$$

where index family $\mathcal{E}$ on $X^{0}$ reads as

$$
\mathcal{E}\left(X_{j}\right)=\left\{\left(\eta+\sum_{i} e_{f}\left(X_{j}, Y_{i}\right) z_{i}, \sum_{i} q_{i}\right) \mid\left(z_{i}, q_{i}\right) \in \mathcal{E}^{0}\left(Y_{i}\right), \eta \in \mathbb{Z}_{+}\right\},
$$

the sum is taken over all $i=1, \ldots, r_{0}$ such that $e_{f}\left(X_{j}, Y_{i}\right) \neq 0$.

The proof of Theorem 5 will be given in Appendix A.

4.2. Push-forwards. Let us recall the definitions of the push-forward operator associated with a map of smooth manifolds.

We denote

$$
\mathcal{D}^{\prime}(Y, G)=C_{0}^{\infty}\left(Y, G^{*}\right)^{\prime}
$$

The inclusion

$$
C_{0}^{\infty}\left(Y, G \otimes \Omega_{Y}\right) \subset \mathcal{D}^{\prime}(Y, G)
$$

holds true. For each $u \in C_{0}^{\infty}\left(Y, G \otimes \Omega_{Y}\right)$ given by $u=s \otimes \mu$, where $s \in C_{0}^{\infty}(Y, G), \mu \in C_{0}^{\infty}\left(Y, \Omega_{Y}\right)$, the corresponding functional on $C_{0}^{\infty}\left(Y, G^{*}\right)$ is defined by the formula

$$
\langle u, \varphi\rangle=\int_{Y}\langle s(y), \varphi(y)\rangle \mu(y) \in \mathbb{C}, \quad \varphi \in C_{0}^{\infty}\left(Y, G^{*}\right),
$$

where $\langle s(y), \varphi(y)\rangle \in \mathbb{C}$ denotes a value of functional $\varphi(y) \in G_{y}^{*}$ on $s(y) \in G_{y}$.

Definition 12. Let $X, Y$ be compact smooth manifolds, $G$ be a vector bundle on $Y$. Given a smooth map $f: X \rightarrow Y$, the push-forward operator is a linear operator

$$
f_{*}: \mathcal{D}^{\prime}\left(X, f^{*} G\right) \rightarrow \mathcal{D}^{\prime}(Y, G)
$$

defined for each $\mu \in \mathcal{D}^{\prime}\left(X, f^{*} G\right)$ by

$$
\left\langle f_{*} \mu, \varphi\right\rangle=\left\langle\mu, f^{*} \varphi\right\rangle, \quad \varphi \in C^{\infty}\left(Y, G^{*}\right) .
$$

Let $X, Y$ be compact smooth manifolds of dimension $n$ and $m$ respectively, $X^{0}=X_{1} \cup \ldots \cup X_{r}$ and $Y^{0}=Y_{1} \cup \ldots \cup Y_{r^{0}}$ be stratified submanifolds of $X$ and $Y$, respectively.

Definition 13. A smooth map $f: X \rightarrow Y$ is said to be a relative fibration if it satisfies the following conditions:

1. $f$ is a relative map;

2. $f$ is surjective;

3. For each component $X_{j}$ of $X^{0}$ there exists at most one component $Y_{i}$ of $Y^{0}$ such that $e_{f}\left(X_{j}, Y_{i}\right) \neq 0$ 
4. Let $p \in X^{0}$ be such that $f(p)=p_{0} \notin Y^{0}$. To be specific we suppose that $p \in X_{1} \bigcap \ldots \bigcap X_{\ell}$ and $p \notin X_{\ell+1} \cup \ldots \cup X_{r}$. As in Definition 11, we write map $f$ in local coordinates:

$$
y_{i}^{0}=f_{i}\left(x, x^{0}\right), \quad\left(x, x^{0}\right) \in \mathbb{R}^{\ell} \times \mathbb{R}^{n-\ell}, \quad i=1, \ldots, m .
$$

Then the rank of Jacobi matrix $\frac{\partial\left(f_{1}, \ldots, f_{m}\right)}{\partial\left(x_{1}^{0}, \ldots, x_{n-\ell}^{0}\right)}$ is equal to $m$.

Theorem 6. Let $\mathcal{E}$ be an index family on $X^{0}$ such that, for each $j=1, \ldots, r$ obeying $e_{f}\left(X_{j}, Y_{i}\right)=0$ for each $i=1, \ldots, r_{0}$, the inequality $\inf \mathcal{E}\left(X_{j}\right)>0$ holds true. Then for each relative fibration $f:\left(X, X^{0}\right) \rightarrow\left(Y, Y^{0}\right)$ and for each line bundle $G$ on $Y$ push-forward operator $f_{*}$ restricts to the operator

$$
f_{*}: \mathcal{A}_{p h g}^{\mathcal{E}}\left(X, X^{0}, f^{*} G \otimes \Omega_{X, X^{0}}\right) \rightarrow \mathcal{A}_{p h g}^{\mathcal{E}^{0}}\left(Y, Y^{0}, G \otimes \Omega_{Y, Y^{0}}\right),
$$

where index family $\mathcal{E}^{0}$ on $Y^{0}$ reads as

$$
\mathcal{E}^{0}\left(Y_{i}\right)=\bar{\bigcup}_{j: e_{f}\left(X_{j}, Y_{i}\right) \neq 0}\left\{\left(\frac{z}{e_{f}\left(X_{j}, Y_{i}\right)}, q\right):(z, q) \in \mathcal{E}\left(X_{j}\right)\right\}, \quad i=1, \ldots, r_{0} .
$$

The proof of Theorem 6 will be given in Appendix $B$.

4.3. Proofs of Theorems 1 and 2. Let us prove Theorem 1. By a straightforward calculation it is easy to check that the map

$$
A: C_{0}^{\infty}\left(Y \backslash Y^{0}, \Omega_{Y}^{\frac{1}{2}}\right) \rightarrow C^{\infty}\left(X \backslash X^{0}, \Omega_{X}^{\frac{1}{2}}\right)
$$

defined by operator $A \in \mathcal{K}^{\mathcal{E}_{1}, \mathcal{E}_{2}}\left(X, X^{0} ; Y, Y^{0}\right)$ can be represented as

$$
A \mu=\pi_{1 *}\left(k_{A} \pi_{2}^{*} \mu\right), \quad \mu \in C_{0}^{\infty}\left(Y \backslash Y^{0}, \Omega_{Y}^{\frac{1}{2}}\right)
$$

where maps $\pi_{1}: X \times Y \rightarrow X, \pi_{2}: X \times Y \rightarrow Y$ are given by

$$
\pi_{1}(x, y)=x ; \quad \pi_{2}(x, y)=y .
$$

Suppose that an index family $\mathcal{F}$ on $Y^{0}$ satisfies the condition $\inf \left(\mathcal{E}_{2}+\mathcal{F}\right)>0$ and $\mu \in$ $\mathcal{A}_{p h g}^{\mathcal{F}}\left(Y, Y^{0}, \Omega_{Y, Y^{0}}^{\frac{1}{2}}\right)$. One can show that $\pi_{2}$ is a relative map and moreover $e_{\pi_{2}}\left(X^{0} \times Y, Y^{0}\right)=0$, $e_{\pi_{2}}\left(X \times Y^{0}, Y^{0}\right)=1$. Therefore, by Theorem 5 we have:

$$
\pi_{2}^{*} \mu \in \mathcal{A}_{p h g}^{0, \mathcal{F}}\left(X \times Y,\left\{X \times Y^{0}\right\} \cup\left\{X^{0} \times Y\right\}, \pi_{2}^{*} \Omega_{Y, Y^{0}}^{\frac{1}{2}}\right) .
$$

By the properties of conormal functions observed in Remark 1, it follows that

$$
k_{A} \pi_{2}^{*} \mu \in \mathcal{A}_{p h g}^{\mathcal{E}_{1}, \mathcal{E}_{2}+\mathcal{F}}\left(X \times Y,\left\{X \times Y^{0}\right\} \cup\left\{X^{0} \times Y\right\}, \Omega_{X \times Y,\left\{X \times Y^{0}\right\} \cup\left\{X^{0} \times Y\right\}}^{\frac{1}{2}} \otimes \pi_{2}^{*} \Omega_{Y, Y^{0}}^{\frac{1}{2}}\right) .
$$

The isomorphism of vector bundles

$$
\Omega_{X \times Y,\left\{X \times Y^{0}\right\} \cup\left\{X^{0} \times Y\right\}}^{\frac{1}{2}} \cong \pi_{1}^{*} \Omega_{X, X^{0}}^{\frac{1}{2}} \otimes \pi_{2}^{*} \Omega_{Y, Y^{0}}^{\frac{1}{2}}
$$

holds true. Hence,

$$
k_{A} \pi_{2}^{*} \mu \in \mathcal{A}_{p h g}^{\mathcal{E}, \mathcal{E}_{2}+\mathcal{F}}\left(X \times Y,\left\{X \times Y^{0}\right\} \cup\left\{X^{0} \times Y\right\}, \pi_{1}^{*} \Omega_{X, X^{0}}^{-\frac{1}{2}} \otimes \Omega_{X \times Y,\left\{X \times Y^{0}\right\} \cup\left\{X^{0} \times Y\right\}}\right) .
$$

Since $\inf \left(\mathcal{E}_{2}+\mathcal{F}\right)>0$ and one can show that $\pi_{1}$ is a relative fibration with $e_{\pi_{1}}\left(X^{0} \times Y, X^{0}\right)=1$, $e_{\pi_{1}}\left(X \times Y^{0}, X^{0}\right)=0$, by applying Theorem 6 with $G=\Omega_{X^{\prime} X^{0}}^{-\frac{1}{2}}, f=\pi_{1}$ we obtain that $A \mu \in$ $\mathcal{A}_{\text {phg }}^{\mathcal{E}_{1}}\left(X, X^{0}, \Omega_{X, X^{0}}^{\frac{1}{2}}\right)$. It completes the proof of Theorem 1 .

Theorem 2 can be proved in a similar way. The kernel of the composition $C=A \circ B$ is represented as

$$
k_{C}=\pi_{2 *}\left(\pi_{3}^{*} k_{A} \pi_{1}^{*} k_{B}\right),
$$


where the maps $\pi_{1}: X \times Y \times Z \rightarrow Y \times Z, \pi_{2}: X \times Y \times Z \rightarrow X \times Z, \pi_{3}: X \times Y \times Z \rightarrow X \times Y$ are defined by

$$
\pi_{1}(x, y, z)=(y, z) ; \quad \pi_{2}(x, y, z)=(x, z) ; \quad \pi_{3}(x, y, z)=(x, y) .
$$

Now it remains to apply Theorems 5 and 6 .

4.4. Proof of Theorem 3. Let $X$ be a compact smooth manifold with a distinguished submanifold $X^{0}$ of codimension 1 . We suppose that $X$ is equipped with a Riemannian metric $g_{X}$ and the normal bundle of $X^{0}$ is trivial.

We will use the stretched product $X_{b}^{2}$ obtained from $X \times X$ by the blow-up of the submanifold $X^{0} \times X^{0} \subset X \times X$. Let us recall its definition. First of all, we introduce the normal bundle $N\left(X^{0} \times X^{0}\right)=T(X \times X) / T\left(X^{0} \times X^{0}\right)$ of $X^{0} \times X^{0}$. We observe that rank $N\left(X^{0} \times X^{0}\right)=2$.

The projectivization of the bundle $N\left(X^{0} \times X^{0}\right)$ is the bundle $P\left(N\left(X^{0} \times X^{0}\right)\right)$ over $X^{0} \times X^{0}$, whose fiber at $p \in X^{0} \times X^{0}$ consists of one-dimensional linear subspaces in $N_{p}\left(X^{0} \times X^{0}\right)$. We define the set

$$
V\left(N\left(X^{0} \times X^{0}\right)\right)=\bigsqcup_{\ell \in P\left(N\left(X^{0} \times X^{0}\right)\right)} V(\ell),
$$

where $V(\ell) \subset N\left(X^{0} \times X^{0}\right)$ is a one-dimensional linear space corresponding to a line $\ell$. Thus, elements of $V\left(N\left(X^{0} \times X^{0}\right)\right)$ are collections $\left(x_{1}^{0}, x_{2}^{0}, \ell, v\right)$, where $p=\left(x_{1}^{0}, x_{2}^{0}\right) \in X^{0} \times X^{0}, \ell \subset$ $N_{p}\left(X^{0} \times X^{0}\right), v \in V(\ell)$. One can prove that set $V\left(N\left(X^{0} \times X^{0}\right)\right)$ has a structure of smooth manifold. We introduce a map $\beta_{N}: V\left(N\left(X^{0} \times X^{0}\right)\right) \rightarrow N\left(X^{0} \times X^{0}\right)$ by the formula

$$
\beta_{N}:\left(x_{1}^{0}, x_{2}^{0}, \ell, v\right) \mapsto\left(x_{1}^{0}, x_{2}^{0}, v\right) .
$$

Let $g_{X \times X}$ be the Riemannian metric on $X \times X$ coinciding with metric $g_{X}$ on $T X \times\{0\}$ and on $\{0\} \times T X$, which are subsets of $T X \times T X=T(X \times X)$. Moreover, the sets $T X \times\{0\}$ and $\{0\} \times T X$ are mutually orthogonal.

By Proposition 1, there exists a neighborhood $U$ of $X^{0} \times X^{0}$ in $N\left(X^{0} \times X^{0}\right)$ such that the following map is a diffeomorphism:

$$
\left.\exp _{X \times X}\right|_{U}: U \stackrel{\sim}{\rightarrow} \exp (U) .
$$

We introduce an equivalence relation on $\left[(X \times X) \backslash\left(X^{0} \times X^{0}\right)\right] \sqcup \beta_{N}^{-1}(U)$ letting points $\left(p_{1}, p_{2}\right) \in(X \times X) \backslash\left(X^{0} \times X^{0}\right)$ and $\left(x_{1}^{0}, x_{2}^{0}, \ell, v\right) \in \beta_{N}^{-1}(U)$ to be equivalent if and only if $\left(p_{1}, p_{2}\right) \in \exp (U)$ and the identity $\exp \left(\beta_{N}\left(x_{1}^{0}, x_{2}^{0}, \ell, v\right)\right)=\left(p_{1}, p_{2}\right)$ holds true.

The stretched product $X_{b}^{2}$ is defined as the set of equivalence classes on $\left[(X \times X) \backslash\left(X^{0} \times\right.\right.$ $\left.\left.X^{0}\right)\right] \sqcup \beta_{N}^{-1}(U)$ :

$$
X_{b}^{2}=\left[(X \times X) \backslash\left(X^{0} \times X^{0}\right)\right] \sqcup \beta_{N}^{-1}(U) / \sim,
$$

Set $X_{b}^{2}$ is naturally endowed with a structure of smooth manifold.

Let us define a map $\beta: X_{b}^{2} \rightarrow X \times X$ as follows: if $\left(p_{1}, p_{2}\right) \in(X \times X) \backslash\left(X^{0} \times X^{0}\right)$, then

$$
\beta\left(p_{1}, p_{2}\right)=\left(p_{1}, p_{2}\right)
$$

if $\left(x_{1}^{0}, x_{2}^{0}, \ell, v\right) \in \beta_{N}^{-1}(U)$, then

$$
\beta\left(x_{1}^{0}, x_{2}^{0}, \ell, v\right)=\exp \left(\beta_{N}\left(x_{1}^{0}, x_{2}^{0}, \ell, v\right)\right) .
$$

There is a submanifold in $X_{b}^{2}$ :

$$
X_{O b}^{2}=\left\{\left(x_{1}^{0}, x_{2}^{0}, \ell, v\right) \in \beta_{N}^{-1}(U): v \equiv 0\right\} .
$$

We let

$$
X_{1 b}^{2}=X^{0} \times\left(X \backslash X^{0}\right) \sqcup\left\{\left(x_{1}^{0}, x_{2}^{0}, \ell, v\right) \in \beta_{N}^{-1}(U): \ell=\ell_{1}\right\},
$$

where $\ell_{1}$ is the one-dimensional subspace in $N\left(X^{0} \times X^{0}\right)$ consisting of vectors $\left(v_{1}, v_{2}\right) \in T X \times T X$ such that $v_{1} \in T X^{0}$. In the same way we define

$$
X_{2 b}^{2}=\left(X \backslash X^{0}\right) \times X^{0} \sqcup\left\{\left(x_{1}^{0}, x_{2}^{0}, \ell, v\right) \in \beta_{N}^{-1}(U): \ell=\ell_{2}\right\},
$$


where $\ell_{2}$ is the one-dimensional subspace in $N\left(X^{0} \times X^{0}\right)$ consisting of vectors $\left(v_{1}, v_{2}\right) \in T X \times T X$ such that $v_{2} \in T X^{0}$.

It is easy to see that $X_{1 b}^{2}, X_{2 b}^{2}$ and $X_{O b}^{2}$ are smooth submanifolds in $X_{b}^{2}$. These submanifolds intersect transversally, and their union is a stratified submanifold $\mathcal{X}_{b}^{2}$ of manifold $X_{b}^{2}$.

A fundamental property of $X_{b}^{2}$ is provided in the following statement.

Lemma 1. An operator $A$ belongs to class $\mathcal{K}^{\mathcal{E}_{1}, \mathcal{E}_{2}, \mathcal{E}_{O}}\left(X, X^{0}\right)$ if and only if the push-forward $\tilde{k}_{A}=\beta^{*} k_{A}$ of the kernel $k_{A}$ under map $\beta: X_{b}^{2} \rightarrow X \times X$ is a conormal function on $X_{b}^{2}$ with respect to the index family $\left(\mathcal{E}_{1}, \mathcal{E}_{2}, \mathcal{E}_{O}\right)$ on $\mathcal{X}_{b}^{2}=X_{1 b}^{2} \cup X_{2 b}^{2} \cup X_{O b}^{2}$.

We prove Theorem 3 by using Lemma 1 . We introduce the maps $\beta_{1}: X_{b}^{2} \rightarrow X, \beta_{2}: X_{b}^{2} \rightarrow X$ by $\beta_{1}=\pi_{1} \circ \beta, \beta_{2}=\pi_{2} \circ \beta$, where $\pi_{1}$ and $\pi_{2}$ are defined in (6). It can be shown by straightforward calculations that operator $A \in \mathcal{K}^{\mathcal{E}_{1}, \mathcal{E}_{2}, \mathcal{E}_{O}}\left(X, X^{0}\right)$ can be represented as

$$
A \mu=\beta_{1_{*}}\left(\tilde{k}_{A} \beta_{2}^{*} \mu\right), \quad \mu \in C_{0}^{\infty}\left(Y \backslash Y^{0}, \Omega_{Y}^{\frac{1}{2}}\right),
$$

where $\tilde{k}_{A}$ is defined in Lemma 1. Now the proof of Theorem 3 can be completed in the same way as the proof of Theorem 1 by employing Theorems 5 and 6 .

4.5. Proof of Theorem 4. We prove Theorem 4 as follows. First, we define a manifold $X_{b}^{3}$, which is the blow-up of the stratified submanifold $X^{0}=\left(X \times X^{0} \times X^{0}\right) \cup\left(X^{0} \times X \times X^{0}\right) \cup\left(X^{0} \times\right.$ $\left.X^{0} \times X\right)$ in $X \times X \times X$. Then we introduce the maps $\gamma_{i}: X_{b}^{3} \rightarrow X_{b}^{2}, i=1,2,3$ being analogues of projections $\pi_{i}, i=1,2,3$ (cf. (7)). One can show that the kernel $k_{C}$ of the composition can be represented in the form

$$
k_{C}=\gamma_{2 *}\left(\gamma_{3}^{*} k_{A} \gamma_{1}^{*} k_{B}\right),
$$

where $\gamma_{3}^{*} k_{A}, \gamma_{1}^{*} k_{B}$ are the lifts of the kernels to $X_{b}^{3}$. An important fact is the statement that there exists a stratified submanifold $\mathcal{X}_{b}^{3}$ in $X_{b}^{3}$ such that maps $\gamma_{i}:\left(X_{b}^{3}, \mathcal{X}_{b}^{3}\right) \rightarrow\left(X_{b}^{2}, \mathcal{X}_{b}^{2}\right)$ are relative fibrations. Then the proof is completed by using Theorems 5 and 6 .

Let us describe the constructions of manifold $X_{b}^{3}$, submanifold $\mathcal{X}_{b}^{3}$ and maps $\gamma_{i}$. We consider the normal bundle $N\left(X^{0} \times X^{0} \times X^{0}\right)=T(X \times X \times X) / T\left(X^{0} \times X^{0} \times X^{0}\right)$ of the submanifold $X^{0} \times X^{0} \times X^{0}$ of rank 3. The projectivization of bundle $N\left(X^{0} \times X^{0} \times X^{0}\right)$ is the bundle $P\left(N\left(X^{0} \times X^{0} \times X^{0}\right)\right)$ over $X^{0} \times X^{0} \times X^{0}$, whose fiber at $p \in X^{0} \times X^{0} \times X^{0}$ consists of one-dimensional linear subspaces in $N_{p}\left(X^{0} \times X^{0} \times X^{0}\right)$. We introduce the set

$$
V\left(N\left(X^{0} \times X^{0} \times X^{0}\right)\right)=\bigsqcup_{\ell \in P\left(N\left(X^{0} \times X^{0} \times X^{0}\right)\right)} V(\ell)
$$

where $V(\ell) \subset N\left(X^{0} \times X^{0} \times X^{0}\right)$ is the one-dimensional linear subspace corresponding to $\ell$ as an element of $N\left(X^{0} \times X^{0} \times X^{0}\right)$. Thus, elements of $V\left(N\left(X^{0} \times X^{0} \times X^{0}\right)\right)$ are collections $(p, \ell, v)$, where $p \in X^{0} \times X^{0} \times X^{0}, \ell \subset N_{p}\left(X^{0} \times X^{0} \times X^{0}\right)$ and $v \in V(\ell)$. It is easy to show that the set $V\left(N\left(X^{0} \times X^{0} \times X^{0}\right)\right)$ has a structure of a smooth manifold.

We define a submanifold $V_{0}$ in $V\left(N\left(X^{0} \times X^{0} \times X^{0}\right)\right)$ by

$$
V_{0}=\left\{(p, \ell, v) \in V\left(N\left(X^{0} \times X^{0} \times X^{0}\right)\right): v=0\right\} .
$$

We introduce a map $\gamma_{N}: V\left(N\left(X^{0} \times X^{0} \times X^{0}\right)\right) \rightarrow N\left(X^{0} \times X^{0} \times X^{0}\right)$ by the formula

$$
\gamma_{N}:\left(x_{1}^{0}, x_{2}^{0}, x_{3}^{0}, \ell, v\right) \mapsto\left(x_{1}^{0}, x_{2}^{0}, x_{3}^{0}, v\right) .
$$

It is easy to show that the restriction of $\gamma_{N}$ to $V \backslash V_{0}$ defines the diffeomorphism

$$
\left.\gamma_{N}\right|_{V \backslash V_{0}}: V\left(N\left(X^{0} \times X^{0} \times X^{0}\right)\right) \backslash V_{0} \stackrel{\sim}{\rightarrow} N\left(X^{0} \times X^{0} \times X^{0}\right) \backslash\left(X^{0} \times X^{0} \times X^{0}\right) .
$$

As in the two-dimensional case, one can introduce the notion of blow-up of submanifolds $\widehat{X}_{1}=X \times X^{0} \times X^{0}, \widehat{X}_{2}=X^{0} \times X \times X^{0}$ and $\widehat{X}_{3}=X^{0} \times X^{0} \times X$ of the manifold $X \times X \times X$. 
We consider the normal bundle $N\left(\widehat{X}_{1}\right)$ of submanifold $\widehat{X}_{1}$, whose fiber at $p \in \widehat{X}_{1}$ is $N_{p}\left(\widehat{X}_{1}\right)=$ $T_{p}(X \times X \times X) / T_{p}\left(\widehat{X}_{1}\right)$ for each $p=\left(x_{1}, x_{2}^{0}, x_{3}^{0}\right) \in \widehat{X}_{1}$. The map

$$
p r_{1}: N\left(\widehat{X}_{1}\right) \rightarrow N\left(X^{0} \times X^{0}\right),\left(x_{1}, x_{2}^{0}, x_{3}^{0}, v_{1}\right) \mapsto\left(x_{2}^{0}, x_{3}^{0}, v_{1}\right),
$$

defines an isomorphism $N_{p}\left(\widehat{X}_{1}\right) \cong N_{\left(x_{2}^{0}, x_{3}^{0}\right)}\left(X^{0} \times X^{0}\right)$.

We introduce the bundle $P\left(N\left(\widehat{X}_{1}\right)\right)$ over $\widehat{X}_{1}$, whose fiber at $p=\left(x_{1}, x_{2}^{0}, x_{3}^{0}\right) \in \widehat{X}_{1}$ consists of one-dimensional linear subspaces in $N_{p}\left(\widehat{X}_{1}\right)$. We define the set

$$
V\left(N\left(\widehat{X}_{1}\right)\right)=\bigsqcup_{\ell \in P\left(N\left(\widehat{X}_{1}\right)\right)} V(\ell)
$$

where $V(\ell) \subset N\left(\widehat{X}_{1}\right)$ is the one-dimensional linear subspace corresponding to $\ell$ as an element of $N\left(\widehat{X}_{1}\right)$. Thus, elements of $V\left(N\left(\widehat{X}_{1}\right)\right)$ are collections $(p, \ell, v)$, where $p=\left(x_{1}, x_{2}^{0}, x_{3}^{0}\right) \in \widehat{X}_{1}$, $\ell \subset N_{p}\left(\widehat{X}_{1}\right)$ and $v \in V(\ell)$.

We define the map

$$
\gamma_{N_{1}}: V\left(N\left(\widehat{X}_{1}\right)\right) \rightarrow N\left(\widehat{X}_{1}\right),\left(x_{1}, x_{2}^{0}, x_{3}^{0}, \ell_{1}, v_{1}\right) \mapsto\left(x_{1}, x_{2}^{0}, x_{3}^{0}, v_{1}\right) .
$$

Similar objects can be introduced for the submanifolds $\widehat{X}_{2}$ and $\widehat{X}_{3}$. In particular, there are defined maps $\gamma_{N_{i}}: V\left(N\left(\widehat{X}_{i}\right)\right) \rightarrow N\left(\widehat{X}_{i}\right)$ and $p r_{i}: N\left(\widehat{X}_{i}\right) \rightarrow N\left(X^{0} \times X^{0}\right), i=2,3$.

We introduce the submanifold $V_{i}$ in $V\left(N\left(\widehat{X}_{i}\right)\right), i=1,2,3$, by the formula

$$
V_{i}=\left\{(p, \ell, v) \in V\left(N\left(\widehat{X}_{i}\right)\right): v=0\right\} .
$$

It is easy to show that the restriction of $\gamma_{N_{i}}$ to $V \backslash V_{i}, i=1,2,3$, defines the diffeomorphism

$$
\left.\gamma_{N_{1}}\right|_{V \backslash V_{i}}: V\left(N\left(\widehat{X}_{i}\right)\right) \backslash V_{i} \stackrel{\sim}{\rightarrow} N\left(\widehat{X}_{i}\right) \backslash \widehat{X}_{i} .
$$

Let $g_{X \times X \times X}$ be the Riemannian metric on $X \times X \times X$ coinciding with metric $g_{X}$ on subbundles $T X \times\{0\} \times\{0\},\{0\} \times T X \times\{0\},\{0\} \times\{0\} \times T X$ of $T X \times T X \times T X=T(X \times X \times X)$. By Proposition 1, there exists a neighborhood $U$ of $X^{0} \times X^{0} \times X^{0}$ in $N\left(X^{0} \times X^{0} \times X^{0}\right)$ such that the map

$$
\exp :=\left.\exp _{X \times X \times X}\right|_{U}: U \stackrel{\sim}{\rightarrow} \exp _{X \times X \times X}(U) \subset X \times X \times X
$$

is a diffeomorphism, as well as there exists a neighborhood $U_{1}$ of $X^{0} \times X^{0}$ in $N\left(X^{0} \times X^{0}\right)$ such that the map

$$
\left.\exp _{X \times X}\right|_{U_{1}}: U_{1} \stackrel{\sim}{\rightarrow} \exp _{X \times X}\left(U_{1}\right) \subset X \times X
$$

is a diffeomorphism. For each $i=1,2,3$, the composition of map $\exp _{X \times X}$ with $p r_{i}$ is a diffeomorphism

$$
\exp _{i}: p r_{i}^{-1}\left(U_{1}\right) \subset N\left(\widehat{X}_{i}\right) \stackrel{\sim}{\rightarrow} \exp _{i}\left(p r_{i}^{-1}\left(U_{1}\right)\right) \subset X \times X \times X .
$$

We introduce an equivalence relation $\sim$ on $\left(X \times X \times X \backslash \widehat{X}^{0}\right) \sqcup \gamma_{N}^{-1}(U) \sqcup \gamma_{N_{1}}^{-1}\left(U_{1}\right) \sqcup \gamma_{N_{2}}^{-1}\left(U_{1}\right) \sqcup$ $\gamma_{N_{3}}^{-1}\left(U_{1}\right)$ letting

- Points $\left(p_{1}, p_{2}, p_{3}\right) \in X \times X \times X \backslash \widehat{X}^{0}$ and $\left(x_{1}^{0}, x_{2}^{0}, x_{3}^{0}, \ell, v\right) \in \gamma_{N}^{-1}(U)$ are equivalent if and only if $\left(p_{1}, p_{2}, p_{3}\right) \in \exp (U)$ and

$$
\exp \left(\gamma_{N}\left(x_{1}^{0}, x_{2}^{0}, x_{3}^{0}, \ell, v\right)\right)=\left(p_{1}, p_{2}, p_{3}\right)
$$

- For each $i=1,2,3$, points $\left(p_{1}, p_{2}, p_{3}\right) \in X \times X \times X \backslash \widehat{X}^{0}$ and $\left(p, \ell_{1}, v_{1}\right) \in \gamma_{N_{i}}^{-1}\left(U_{1}\right)$ are equivalent if and only if $\left(p_{1}, p_{2}, p_{3}\right) \in \exp _{i}\left(\operatorname{pr}_{i}^{-1}\left(U_{1}\right)\right)$ and

$$
\exp _{i}\left(\gamma_{N_{i}}\left(p, \ell_{1}, v_{1}\right)\right)=\left(p_{1}, p_{2}, p_{3}\right)
$$


- For each $i=1,2,3$, points $\left(x_{1}^{0}, x_{2}^{0}, x_{3}^{0}, \ell, v\right) \in \gamma_{N}^{-1}(U)$ and $\left(p, \ell_{1}, v_{1}\right) \in \gamma_{N_{i}}^{-1}\left(U_{1}\right)$ are equivalent if and only if

$$
\left(x_{1}^{0}, x_{2}^{0}, x_{3}^{0}\right)=p,
$$

and $(\ell, v)$ is mapped to $\left(\ell_{1}, v_{1}\right)$ under the natural map $N(X) \rightarrow N\left(\widehat{X}_{i}\right)$.

We define set $X_{b}^{3}$ as the set of equivalence classes:

$$
X_{b}^{3}=\left(X \times X \times X \backslash \widehat{X}^{0}\right) \sqcup \gamma_{N}^{-1}(U) \sqcup \gamma_{N_{1}}^{-1}\left(U_{1}\right) \sqcup \gamma_{N_{2}}^{-1}\left(U_{1}\right) \sqcup \gamma_{N_{3}}^{-1}\left(U_{1}\right) / \sim .
$$

It is easy to check that $X_{b}^{3}$ is a smooth manifold.

We introduce the following subsets in $X_{b}^{3}$ :

$$
\begin{aligned}
& X_{0}^{3}=\left\{(p, \ell, v) \in V\left(N\left(X^{0} \times X^{0} \times X^{0}\right)\right): v=0\right\} \subset \gamma_{N}^{-1}(U), \\
& X_{O i}^{3}=\left\{(p, \ell, v) \in V\left(N\left(\widehat{X}_{i}\right)\right): v=0\right\} \subset \gamma_{N_{i}}^{-1}\left(U_{1}\right), \quad i=1,2,3 .
\end{aligned}
$$

We define subset $X_{1}^{3}$ in $X_{b}^{3}$ by its intersections with the components of $X_{b}^{3}$ :

$$
\begin{aligned}
& X_{1}^{3} \cap\left(X \times X \times X \backslash \widehat{X}^{0}\right)=X^{0} \times\left(X \backslash X^{0}\right) \times\left(X \backslash X^{0}\right), \\
& X_{1}^{3} \cap \gamma_{N}^{-1}(U)=\left\{(p, \ell, v) \in V\left(N\left(X^{0} \times X^{0} \times X^{0}\right)\right): \ell \subset T X^{0} \times T X \times T X\right\}, \\
& X_{1}^{3} \cap \gamma_{N_{1}}^{-1}\left(U_{1}\right)=\left\{(p, \ell, v) \in V\left(N\left(\widehat{X}_{1}\right)\right): p \in X^{0} \times X^{0} \times X^{0}\right\}, \\
& X_{1}^{3} \cap \gamma_{N_{2}}^{-1}\left(U_{1}\right)=\left\{(p, \ell, v) \in V\left(N\left(\widehat{X}_{2}\right)\right): \ell \subset T X^{0} \times T X \times T X\right\}, \\
& X_{1}^{3} \cap \gamma_{N_{3}}^{-1}\left(U_{1}\right)=\left\{(p, \ell, v) \in V\left(N\left(\widehat{X}_{3}\right)\right): \ell \subset T X^{0} \times T X \times T X\right\} .
\end{aligned}
$$

In the same way we define subsets $X_{2}^{3}$ and $X_{3}^{3}$.

It is easy to see that all the subsets introduced above are smooth submanifolds in $X_{b}^{3}$. These submanifolds intersect transversally, and their union is a stratified submanifold in $X_{b}^{3}$, which we denote by $\mathcal{X}_{b}^{3}$ :

$$
\mathcal{X}_{b}^{3}=X_{0}^{3} \cup X_{1}^{3} \cup X_{2}^{3} \cup X_{3}^{3} \cup X_{O 1}^{3} \cup X_{O 2}^{3} \cup X_{O 3}^{3} .
$$

Maps $\gamma_{i}: X_{b}^{3} \rightarrow X_{b}^{2}, i=1,2,3$, are defined as follows.

For $\left(p_{1}, p_{2}, p_{3}\right) \in X \times X \times X \backslash \widehat{X}^{0}$

$$
\gamma_{i}\left(p_{1}, p_{2}, p_{3}\right)=\pi_{i}\left(p_{1}, p_{2}, p_{3}\right),
$$

where maps $\pi_{i}: X \times X \times X \rightarrow X \times X$ are defined by (7).

For $\left(x_{1}^{0}, x_{2}^{0}, x_{3}^{0}, \ell, v\right) \in \gamma_{N}^{-1}(U)$

$$
\begin{aligned}
& \gamma_{1}:\left(x_{1}^{0}, x_{2}^{0}, x_{3}^{0}, \ell, v\right) \mapsto\left(x_{2}^{0}, x_{3}^{0}, \ell_{1}, v_{2}, v_{3}\right), \\
& \gamma_{2}:\left(x_{1}^{0}, x_{2}^{0}, x_{3}^{0}, \ell, v\right) \mapsto\left(x_{1}^{0}, x_{3}^{0}, \ell_{2}, v_{1}, v_{3}\right), \\
& \gamma_{3}:\left(x_{1}^{0}, x_{2}^{0}, x_{3}^{0}, \ell, v\right) \mapsto\left(x_{1}^{0}, x_{2}^{0}, \ell_{3}, v_{1}, v_{2}\right),
\end{aligned}
$$

where $\ell_{1}, \ell_{2}, \ell_{3}$ are the images of $\ell$ under the projections $N\left(X^{0} \times X^{0} \times X^{0}\right)$ on $N\left(X^{0} \times X^{0}\right)$ : $\left(x_{1}^{0}, x_{2}^{0}, x_{3}^{0}, v\right) \mapsto\left(x_{2}^{0}, x_{3}^{0}, v_{2}, v_{3}\right),\left(x_{1}^{0}, x_{2}^{0}, x_{3}^{0}, v\right) \mapsto\left(x_{1}^{0}, x_{3}^{0}, v_{1}, v_{3}\right),\left(x_{1}^{0}, x_{2}^{0}, x_{3}^{0}, v\right) \mapsto\left(x_{1}^{0}, x_{2}^{0}, v_{1}, v_{2}\right)$ respectively.

For $(p, \ell, v) \in \gamma_{N_{1}}^{-1}\left(U_{1}\right)$, where $p=\left(x_{1}, x_{2}^{0}, x_{3}^{0}\right) \in \widehat{X}_{1}, \ell \subset N_{p}\left(\widehat{X}_{1}\right)$ and $v \in V(\ell)$, we let

$$
\begin{aligned}
& \gamma_{1}:\left(x_{1}, x_{2}^{0}, x_{3}^{0}, \ell, v\right) \mapsto\left(x_{2}^{0}, x_{3}^{0}, \operatorname{pr}_{1}(\ell), \operatorname{pr}_{1}(v)\right), \\
& \gamma_{2}:\left(x_{1}, x_{2}^{0}, x_{3}^{0}, \ell, v\right) \mapsto\left(x_{1}, \exp _{X}\left(v_{3}\right)\right), \\
& \gamma_{3}:\left(x_{1}, x_{2}^{0}, x_{3}^{0}, \ell, v\right) \mapsto\left(x_{1}, \exp _{X}\left(v_{2}\right)\right) .
\end{aligned}
$$

For $(p, \ell, v) \in \gamma_{N_{i}}^{-1}\left(U_{1}\right)$, maps $\gamma_{1}, \gamma_{2}, \gamma_{3}$ are defined in the same way. 


\section{REGULARIZED TRACE}

Operators in class $\mathcal{K}^{\mathcal{E}_{1}, \mathcal{E}_{2}, \mathcal{E}_{O}}\left(X, X^{0}\right)$ are not, in general, of trace class. It turns out that, if index set $\mathcal{E}_{O}$ satisfies the condition:

$$
\inf \mathcal{E}_{O} \geqslant 0 \text {, and, in addition, if }(0, q) \in \mathcal{E}_{O} \text {, then } q=0,
$$

one can introduce a functional on $\mathcal{K}^{\mathcal{E}_{1}, \mathcal{E}_{2}, \mathcal{E}_{O}}\left(X, X^{0}\right)$ called the regularized trace functional, which coincides with the trace functional on trace class operators.

Before giving the definition of the regularized trace, we introduce the notion of regularized integral for conormal densities.

5.1. Regularized integral. Let $\mu$ be a density defined on a compact manifold $X$ with a distinguished smooth submanifold $X^{0}$ of codimension 1 and this density is conormal with respect to an index family $\mathcal{E}$ and. We suppose that the conormal bundle of $X^{0}$ is trivial, and index family $\mathcal{E}$ satisfies the condition $(9)$. We fix a Riemannian metric $g_{X}$ on $X$ and define continuous function $r$ on $X$ by $r(p)=\varrho\left(p, X^{0}\right)$, where $\varrho$ is the geodesic distance from $p$ to submanifold $X^{0}$.

Definition 14. The regularized integral of density $\mu$ over $X$ is defined by the formula

$$
\int_{X}^{r} \mu=\lim _{\varepsilon \rightarrow 0}\left(\int_{\substack{X \\ r(p)>\varepsilon}} \mu+\left.2 \ln \varepsilon \int_{X^{0}} \mu\right|_{X^{0}}\right) .
$$

Here $\left.\mu\right|_{X^{0}}$ is a density on $X^{0}$ defined as follows. In the normal coordinate system $\exp (U) \rightarrow$ $(-\varepsilon, \varepsilon) \times X^{0}, p \mapsto\left(x, x^{0}\right)$ near $X^{0}$, we write $\mu=u\left(x, x^{0}\right)\left|\frac{d x}{x} d x^{0}\right|$, where $u$ is a conormal function on $(-\varepsilon, \varepsilon) \times X^{0}$ with distinguished submanifold $\{0\} \times \stackrel{x}{X}^{0},\left|d x^{0}\right|$ is the fixed smooth density on $X^{0}$. Since index family $\mathcal{E}$ satisfies $(9)$, it is easy to see that $u$ is extended to a continuous function on $(-\varepsilon, \varepsilon) \times X^{0}$. We let

$$
\left.\mu\right|_{X^{0}}=u\left(0, x^{0}\right)\left|d x^{0}\right|
$$

It is easy to check that $\left.\mu\right|_{X^{0}}$ is independent of the choice of density $\left|d x^{0}\right|$.

One can show that the limit at the right-hand side of 10 exists. One should note that the regularized integral depends on the choice of Riemannian metric $g_{X}$.

5.2. Regularized trace. Let $X$ be a compact manifold and $A: C^{\infty}\left(X, \Omega_{X}^{\frac{1}{2}}\right) \rightarrow C^{\infty}\left(X, \Omega_{X}^{\frac{1}{2}}\right)$ be an integral operator with smooth kernel $k_{A} \in C^{\infty}\left(X \times X, \Omega_{X \times X}^{\frac{1}{2}}\right)$, whose action on a halfdensity $\mu \in C^{\infty}\left(X, \Omega^{\frac{1}{2}}\right)$ is given by formula (2). We recall that such an operator $A$ determines a bounded operator in the space $L^{2}\left(X, \Omega_{X}^{\frac{1}{2}}\right)$. This operator is trace class, and

$$
\operatorname{Tr}(A)=\left.\int_{X} k_{A}\right|_{\Delta}
$$

where $\Delta=\{(x, x) \in X \times X: x \in X\}$.

Here smooth density $\left.k_{A}\right|_{\Delta}$ on $X$ is defined as follows. Let $d v_{X}$ be a smooth positive density on $X$. We write

$$
k_{A}=K_{A}\left(p_{1}, p_{2}\right)\left|d v_{X}\left(p_{1}\right)\right|^{\frac{1}{2}}\left|d v_{X}\left(p_{2}\right)\right|^{\frac{1}{2}}, \quad p_{1}, p_{2} \in X,
$$

where $K_{A} \in C^{\infty}(X \times X)$, and let

$$
\left.k_{A}\right|_{\Delta}=K_{A}(p, p)\left|d v_{X}(p)\right| .
$$

It is easy to check that this definition is independent of the choice of density $d v_{X}$.

Let $X$ be a compact manifold, $X^{0}$ be its smooth submanifold of codimension $1, g_{X}$ be a Riemannian metric on $X$. We suppose that the normal bundle of $X^{0}$ is trivial and consider an 
operator $A \in \mathcal{K}^{\mathcal{E}_{1}, \mathcal{E}_{2}, \mathcal{E}_{O}}\left(X, X^{0}\right)$ with kernel $k_{A} \in C^{\infty}\left(X \times X \backslash\left(X^{0} \times X\right) \cup\left(X \times X^{0}\right), \Omega_{X^{2}}^{\frac{1}{2}}\right)$. We assume that index family $\mathcal{E}_{O}$ satisfies $(9)$.

Definition 15. The regularized trace of operator $A$ is defined by the formula

$$
\mathrm{r}-\operatorname{Tr}(A)=\left.\int_{X}^{r} k_{A}\right|_{\Delta}
$$

One can show that $\left.k_{A}\right|_{\Delta}$ is a conormal density on $\left(X, X^{0}\right)$ with respect to index family $\mathcal{E}_{O}$, and, therefore, the regularized integral of $\left.k_{A}\right|_{\Delta}$ over $X$ is well-defined.

5.3. Regularized trace of the commutator. As above, let $X$ be a compact manifold, $X^{0}$ be its smooth submanifold of codimension $1, g_{X}$ be a Riemannian metric on $X$. Suppose that the normal bundle of $X^{0}$ is trivial. The regularized trace functional $\mathrm{r}$ - $\operatorname{Tr}$ on algebra $\mathcal{K}^{\mathcal{E}_{1}, \mathcal{E}_{2}, \mathcal{E}_{O}}\left(X, X^{0}\right)$ is not a trace functional, i.e., the regularized trace $\mathrm{r}-\operatorname{Tr}([A, B])$ of the commutator of operators $A \in \mathcal{K}^{\mathcal{E}_{1}, \mathcal{E}_{2}, \mathcal{E}_{O}}\left(X, X^{0}\right)$ and $B \in \mathcal{K}^{\mathcal{E}_{1}, \mathcal{E}_{2}, \mathcal{E}_{O}}\left(X, X^{0}\right)$, in general, is nonzero. The main result of this section is a formula providing an expression for the regularized trace of the commutator $\mathrm{r}-\operatorname{Tr}([A, B])$ in terms of certain integral operators on submanifold $X^{0}$ associated with $A$ and $B$.

We begin with the de

nition of a class of operators, for which the aforementioned formula holds true.

Definition 16. We say that $A \in \mathcal{K}\left(X, X^{0}\right)$ if $A \in \mathcal{K}^{\mathcal{E}_{1}, \mathcal{E}_{2}, \mathcal{E}_{O}}\left(X, X^{0}\right)$ for some index families $\mathcal{E}_{1}, \mathcal{E}_{2}, \mathcal{E}_{O}$ and the following conditions hold:

1. For each $\varepsilon>0$, there exists $\delta>0$ such that, if $\varrho\left(x, X^{0}\right)>\varepsilon, \varrho\left(y, X^{0}\right)<\delta$ or $\varrho\left(y, X^{0}\right)>\varepsilon$, $\varrho\left(x, X^{0}\right)<\delta$, then $k_{A}(x, y)=0$.

2. $\mathcal{E}_{O}$ satisfies condition (9).

3. Choose a normal coordinate system with coordinates $\left(x, x^{0}\right) \in(-\varepsilon, \varepsilon) \times X^{0}$ in some tubular neighborhood of $X^{0}$. There exist $m, M, 0<m<M<\infty$, such that function $\widetilde{K}_{A}$ defined by (4) is supported in the set of all $\left(x, s, x_{1}^{0}, x_{2}^{0}\right) \in \Pi_{\varepsilon} \times X^{0} \times X^{0}$ such that $m<|s|<M$.

Using Theorem 4 , it is easy to show that $\mathcal{K}\left(X, X^{0}\right)$ is an algebra.

Before we formulate the statement on the regularized trace of the commutator, we introduce the notions of indicial operator and indicial family associated with an operator $A \in \mathcal{K}\left(X, X^{0}\right)$, which we need to formulate this theorem. limit

Condition (2) of Definition 16 implies that, for an operator $A \in \mathcal{K}\left(X, X^{0}\right)$, there exists the

$$
\lim _{x \rightarrow 0} \widetilde{K}_{A}\left(x, s, x_{1}^{0}, x_{2}^{0}\right)=: \widetilde{K}_{A}\left(0, s, x_{1}^{0}, x_{2}^{0}\right),
$$

where $\widetilde{K}_{A}$ is the function given by formula (4).

Definition 17. The indicial operator associated with an operator $A \in \mathcal{K}\left(X, X^{0}\right)$ is the operator

$$
I(A): C_{0}^{\infty}\left((\mathbb{R} \backslash\{0\}) \times X^{0}, \Omega_{\mathbb{R} \backslash\{0\} \times X^{0}}^{\frac{1}{2}}\right) \rightarrow C_{0}^{\infty}\left((\mathbb{R} \backslash\{0\}) \times X^{0}, \Omega_{\mathbb{R} \backslash\{0\} \times X^{0}}^{\frac{1}{2}}\right),
$$

whose action on the half-density

$$
\mu=u\left(x, x^{0}\right)\left|\frac{d x}{x} d x^{0}\right|^{\frac{1}{2}} \in C_{0}^{\infty}\left((\mathbb{R} \backslash\{0\}) \times X^{0}, \Omega_{(R \backslash\{0\}) \times X^{0}}^{\frac{1}{2}}\right)
$$

is

$$
I(A) \mu=I(A) u\left(x, x^{0}\right)\left|\frac{d x}{x} d x^{0}\right|^{\frac{1}{2}}
$$


where

$$
I(A) u\left(x, x^{0}\right)=\int_{X^{0}} \int_{-\infty}^{+\infty} \widetilde{K}_{A}\left(0, s, x^{0}, x_{1}^{0}\right) u\left(\frac{x}{s}, x_{1}^{0}\right) \frac{d s}{s} d x_{1}^{0}, \quad x \in \mathbb{R} \backslash\{0\}, x^{0} \in X^{0} .
$$

The following notion is an analogue of the known notion of the conormal symbol (cf., for instance, [13, 16]) in the situation under consideration.

Definition 18. The indicial families of an operator $A \in \mathcal{K}\left(X, X^{0}\right)$ are the families $\left\{I^{ \pm}(A, \lambda): \lambda \in \mathbb{C}\right\}$ of integral operators on $X^{0}$ with smooth kernels given by:

$$
\begin{aligned}
& K_{I^{+}(A, \lambda)}\left(x_{1}^{0}, x_{2}^{0}\right)=\int_{0}^{+\infty} s^{-i \lambda} \widetilde{K}_{A}\left(0, s, x_{1}^{0}, x_{2}^{0}\right) \frac{d s}{s}, \\
& K_{I^{-}(A, \lambda)}\left(x_{1}^{0}, x_{2}^{0}\right)=\int_{-\infty}^{0}|s|^{-i \lambda} \widetilde{K}_{A}\left(0, s, x_{1}^{0}, x_{2}^{0}\right) \frac{d s}{|s|} .
\end{aligned}
$$

The function $\lambda \mapsto K_{I^{+}(A, \lambda)}\left(x_{1}^{0}, x_{2}^{0}\right)$ (resp. $\lambda \mapsto K_{I^{-}(A, \lambda)}\left(x_{1}^{0}, x_{2}^{0}\right)$ ) is the Mellin transform of function $\widetilde{K}_{A}\left(0, s, x_{1}^{0}, x_{2}^{0}\right)$ (resp. $\widetilde{K}_{A}\left(0,-s, x_{1}^{0}, x_{2}^{0}\right)$ ) with respect to variable $s$ on the semi-axis $(0,+\infty)$. Since $\widetilde{K}_{A}\left(0, s, x_{1}^{0}, x_{2}^{0}\right)$ is a smooth compactly supported function of $s \in(-\infty, 0) \cup$ $(0,+\infty)$ for fixed $x_{1}^{0}, x_{2}^{0} \in X^{0}$, by the Paley-Wiener theorem, functions $K_{I^{ \pm}(A, \lambda)}\left(x_{1}^{0}, x_{2}^{0}\right)$ are well-defined for each $\lambda \in \mathbb{C}$ and are entire functions.

The following properties of the indicial operators hold:

1. $I(A \circ B)=I(A) \circ I(B)$.

2. $I^{+}(A \circ B, \lambda)=I^{+}(A, \lambda) \circ I^{+}(B, \lambda)+I^{-}(A, \lambda) \circ I^{-}(B, \lambda)$.

3. $I^{-}(A \circ B, \lambda)=I^{+}(A, \lambda) \circ I^{-}(B, \lambda)+I^{-}(A, \lambda) \circ I^{+}(B, \lambda)$.

Theorem 7. If $A \in \mathcal{K}\left(X, X^{0}\right)$ and $B \in \mathcal{K}\left(X, X^{0}\right)$, then

$$
\mathrm{r}-\operatorname{Tr}([A, B])=-\frac{1}{\pi i} \int_{-\infty}^{+\infty} \operatorname{tr}\left(\partial_{\lambda} I^{+}(A, \lambda) \circ I^{+}(B, \lambda)+\partial_{\lambda} I^{-}(A, \lambda) \circ I^{-}(B, \lambda)\right) d \lambda,
$$

where the symbol tr stands for the trace of an integral operator on $X^{0}$.

Доказательство. Ву definition we have:

$$
\mathrm{r}-\operatorname{Tr}[(A, B)]=\lim _{\varepsilon \rightarrow 0}\left(\left.\int_{\substack{X \\ r(p)>\varepsilon}}\left(k_{A B}-k_{B A}\right)\right|_{\Delta}+\left.2 \ln \varepsilon \int_{X^{0}}\left(\left.\left(k_{A B}-k_{B A}\right)\right|_{\Delta}\right)\right|_{X^{0}}\right) .
$$

We define map $R: X \times X \rightarrow X \times X$ by $R\left(p_{1}, p_{2}\right)=\left(p_{2}, p_{1}\right)$. Then one can write

$$
\left.\int_{\substack{X \\ r\left(p_{1}\right)>\varepsilon}}\left(k_{A B}\right)\right|_{\Delta}=\int_{\underset{X}{X}}\left(\int_{X} k_{A}\left(p_{1}, p_{2}\right) k_{B}\left(p_{2}, p_{1}\right)\right)=\int_{\substack{X \times X \\ r\left(p_{1}\right)>\varepsilon}} k_{A} R^{*} k_{B}\left(p_{1}, p_{2}\right),
$$

where the last integral should be understood as the integral of the density $k_{A} R^{*} k_{B}$ on $X \times X$ over the set $\left\{\left(p_{1}, p_{2}\right) \in X \times X: r\left(p_{1}\right)>\varepsilon\right\}$. In the same way,

$$
\left.\int_{\substack{X \\ r\left(p_{1}\right)>\varepsilon}}\left(k_{B A}\right)\right|_{\Delta}=\int_{\substack{X \times X \\ r\left(p_{1}\right)>\varepsilon}} k_{B} R^{*} k_{A}\left(p_{1}, p_{2}\right)=\int_{\substack{X \times X \\ r\left(p_{2}\right)>\varepsilon}} k_{A} R^{*} k_{B}\left(p_{1}, p_{2}\right) .
$$


We choose a normal coordinate system with coordinates $\left(x, x^{0}\right) \in\left(-\varepsilon_{1}, \varepsilon_{1}\right) \times X^{0}$ in some tubular neighborhood $V=\exp (U)$ of $X^{0}$. In particular, $V=\left\{p \in X: r(p)<\varepsilon_{1}\right\}$. We obtain that

$$
\begin{aligned}
\left.\int_{\substack{X \\
r\left(p_{1}\right)>\varepsilon}}\left(k_{B A}-k_{B A}\right)\right|_{\Delta}= & \int_{\substack{X \times X \\
r\left(p_{1}\right)>\varepsilon}} k_{A} R^{*} k_{B}\left(p_{1}, p_{2}\right)-\int_{\substack{X \times X \\
r\left(p_{2}\right)>\varepsilon}} k_{A} R^{*} k_{B}\left(p_{1}, p_{2}\right) \\
= & \int_{\substack{V \times V \\
r\left(p_{1}\right)>\varepsilon}} k_{A} R^{*} k_{B}\left(p_{1}, p_{2}\right)-\int_{\substack{V \times V \\
r\left(p_{2}\right)>\varepsilon}} k_{A} R^{*} k_{B}\left(p_{1}, p_{2}\right) \\
& +\int_{\substack{V \times(X \backslash V) \\
r\left(p_{1}\right)>\varepsilon}} k_{A} R^{*} k_{B}\left(p_{1}, p_{2}\right)-\int_{\substack{(X \backslash V) \times V \\
r\left(p_{2}\right)>\varepsilon}} k_{A} R^{*} k_{B}\left(p_{1}, p_{2}\right) \\
& +\int_{\substack{(X \backslash V) \times X \\
r\left(p_{1}\right)>\varepsilon}} k_{A} R^{*} k_{B}\left(p_{1}, p_{2}\right)-\int_{\substack{X \times(X \backslash V) \\
r\left(p_{2}\right)>\varepsilon}} k_{A} R^{*} k_{B}\left(p_{1}, p_{2}\right) .
\end{aligned}
$$

It is easy to see that for each $0<\varepsilon<\varepsilon_{1}$

$$
\begin{aligned}
\int_{\substack{(X \backslash V) \times X \\
r\left(p_{1}\right)>\varepsilon}} k_{A} R^{*} k_{B}\left(p_{1}, p_{2}\right) & =\int_{(X \backslash V) \times X} k_{A} R^{*} k_{B}\left(p_{1}, p_{2}\right), \\
\int_{\substack{X \times(X \backslash V) \\
r\left(p_{2}\right)>\varepsilon}} k_{A} R^{*} k_{B}\left(p_{1}, p_{2}\right) & =\int_{X \times(X \backslash V)} k_{A} R^{*} k_{B}\left(p_{1}, p_{2}\right) .
\end{aligned}
$$

By Condition (1) of Definition 16, there exists $\varepsilon_{2}>0$ such that, if $p_{1} \notin V$ and $r\left(p_{2}\right)<\varepsilon_{2}$ or $r\left(p_{1}\right)<\varepsilon_{2}$ and $p_{2} \notin V$, then $k_{A}\left(p_{1}, p_{2}\right)=k_{B}\left(p_{1}, p_{2}\right)=0$. Hence, for each $0<\varepsilon<\min \left(\varepsilon_{1}, \varepsilon_{2}\right)$

$$
\begin{aligned}
\int_{\substack{(X \backslash V) \times V \\
r\left(p_{2}\right)>\varepsilon}} k_{A} R^{*} k_{B}\left(p_{1}, p_{2}\right) & =\int_{(X \backslash V) \times V} k_{A} R^{*} k_{B}\left(p_{1}, p_{2}\right) . \\
\int_{\substack{V \times(X \backslash V) \\
r\left(p_{1}\right)>\varepsilon}} k_{A} R^{*} k_{B}\left(p_{1}, p_{2}\right) & =\int_{V \times(X \backslash V)} k_{A} R^{*} k_{B}\left(p_{1}, p_{2}\right) .
\end{aligned}
$$

Therefore, we obtain that

$$
\left.\int_{\substack{X \\ r\left(p_{1}\right)>\varepsilon}}\left(k_{B A}-k_{B A}\right)\right|_{\Delta}=\int_{\substack{V \times V \\ r\left(p_{1}\right)>\varepsilon}} k_{A} R^{*} k_{B}\left(p_{1}, p_{2}\right)-\int_{\substack{V \times V \\ r\left(p_{2}\right)>\varepsilon}} k_{A} R^{*} k_{B}\left(p_{1}, p_{2}\right) .
$$

We introduce the local coordinate system $\left(x, s, x_{1}^{0}, x_{2}^{0}\right) \in \Pi_{\varepsilon} \times X^{0} \times X^{0}$ in the neighborhood $\left(V \backslash X^{0}\right) \times\left(V \backslash X^{0}\right)$ given by (3). In these coordinates, map $R$ is written as

$$
R\left(x, s, x_{1}^{0}, x_{2}^{0}\right)=\left(\frac{x}{s}, \frac{1}{s}, x_{2}^{0}, x_{1}^{0}\right) .
$$


Identity 13 becomes

$$
\left.\int_{\substack{X \\ r\left(p_{1}\right)>\varepsilon}}\left(k_{B A}-k_{B A}\right)\right|_{\Delta}=\int_{X^{0} \times X^{0}} \int_{-\infty}^{+\infty}\left(\int_{\varepsilon}^{\varepsilon|s|} \widetilde{K}_{A}\left(x, s, x_{1}^{0}, x_{2}^{0}\right) \widetilde{K}_{B}\left(\frac{x}{s}, \frac{1}{s}, x_{2}^{0}, x_{1}^{0}\right) \frac{d x}{|x|}\right) \frac{d s}{|s|} d x_{1}^{0} d x_{2}^{0},
$$

where functions $\widetilde{K}_{A}$ and $\widetilde{K}_{B}$ are defined by (4).

By Conditions (2) and (3) of Definition 16, it implies easily that the limit

$$
\begin{aligned}
\left.\lim _{\varepsilon \rightarrow 0} \int_{\substack{X \\
r\left(p_{1}\right)>\varepsilon}}\left(k_{B A}-k_{B A}\right)\right|_{\Delta} & =\int_{X^{0} \times X^{0}} \int_{-\infty}^{+\infty}\left(\int_{\varepsilon}^{\varepsilon|s|} \widetilde{K}_{A}\left(0, s, x_{1}^{0}, x_{2}^{0}\right) \widetilde{K}_{B}\left(0, \frac{1}{s}, x_{2}^{0}, x_{1}^{0}\right) \frac{d x}{|x|}\right) \frac{d s}{|s|} d x_{1}^{0} d x_{2}^{0} \\
& =2 \int_{X^{0} \times X^{0}} \int_{-\infty}^{+\infty} \ln |s| \widetilde{K}_{A}\left(0, s, x_{1}^{0}, x_{2}^{0}\right) \widetilde{K}_{B}\left(0, \frac{1}{s}, x_{2}^{0}, x_{1}^{0}\right) \frac{d s}{|s|} d x_{1}^{0} d x_{2}^{0}
\end{aligned}
$$

is well-defined. In particular, it yields

$$
\left.\int_{X^{0}}\left(\left.\left(k_{A B}-k_{B A}\right)\right|_{\Delta}\right)\right|_{X^{0}}=0
$$

Using the relation between the Mellin transform and the Fourier transform and the Parceval identity for the Fourier transform, one can prove that, if $f_{1}, f_{2} \in L^{2}\left((0,+\infty), \frac{d s}{s}\right)$, Mellin transforms $M\left(f_{1}\right), M\left(f_{2}\right)$ belong to $L^{2}(\mathbb{R})$, and we have the formula

$$
\int_{0}^{+\infty} f_{1}(s) \overline{f_{2}(s)} \frac{d s}{s}=\frac{1}{2 \pi} \int_{-\infty}^{+\infty}\left[M\left(f_{1}\right)\right](\lambda) \overline{\left[M\left(f_{2}\right)\right](\lambda)} d \lambda
$$

Applying this formula in the case

$$
f_{1}(s)=\ln |s| \widetilde{K}_{A}\left(0, s, x_{1}^{0}, x_{2}^{0}\right), \quad f_{2}(s)=\overline{\widetilde{K}_{B}\left(0, \frac{1}{s}, x_{2}^{0}, x_{1}^{0}\right)}, \quad s>0
$$

we obtain that

$$
\int_{0}^{+\infty} \ln |s| \widetilde{K}_{A}\left(0, s, x_{1}^{0}, x_{2}^{0}\right) \widetilde{K}_{B}\left(0, \frac{1}{s}, x_{2}^{0}, x_{1}^{0}\right) \frac{d s}{s}=-\frac{1}{2 \pi i} \int_{-\infty}^{+\infty} \partial_{\lambda} K_{I^{+}(A, \lambda)}\left(x_{1}^{0}, x_{2}^{0}\right) K_{I^{+}(B, \lambda)}\left(x_{2}^{0}, x_{1}^{0}\right) d \lambda .
$$

In the same way we have

$$
\int_{-\infty}^{0} \ln |s| \widetilde{K}_{A}\left(0, s, x_{1}^{0}, x_{2}^{0}\right) \widetilde{K}_{B}\left(0, \frac{1}{s}, x_{2}^{0}, x_{1}^{0}\right) \frac{d s}{|s|}=-\frac{1}{2 \pi i} \int_{-\infty}^{+\infty} \partial_{\lambda} K_{I^{-}(A, \lambda)}\left(x_{1}^{0}, x_{2}^{0}\right) K_{I^{-}(B, \lambda)}\left(x_{2}^{0}, x_{1}^{0}\right) d \lambda .
$$


Thus, we obtain that

$$
\begin{aligned}
\operatorname{r}-\operatorname{Tr}([A, B])= & \left.\lim _{\varepsilon \rightarrow 0} \int_{\substack{X \\
r\left(p_{1}\right)>\varepsilon}}\left(k_{B A}-k_{B A}\right)\right|_{\Delta} \\
= & -\frac{1}{\pi i} \int_{X^{0} \times X^{0}} \int_{-\infty}^{+\infty}\left(\partial_{\lambda} K_{I^{+}(A, \lambda)}\left(x_{1}^{0}, x_{2}^{0}\right) K_{I^{+}(B, \lambda)}\left(x_{2}^{0}, x_{1}^{0}\right)\right. \\
& \left.+\partial_{\lambda} K_{I^{-}(A, \lambda)}\left(x_{1}^{0}, x_{2}^{0}\right) K_{I^{-}(B, \lambda)}\left(x_{2}^{0}, x_{1}^{0}\right)\right) d \lambda d x_{1}^{0} d x_{2}^{0} \\
= & -\frac{1}{\pi i} \int_{-\infty}^{+\infty} \operatorname{tr}\left(\partial_{\lambda} I^{+}(A, \lambda) \circ I^{+}(B, \lambda)+\partial_{\lambda} I^{-}(A, \lambda) \circ I^{-}(B, \lambda)\right) d \lambda .
\end{aligned}
$$

\section{A. Proof of Theorem 5}

Let $u \in \mathcal{A}_{\text {phg }}^{\mathcal{E}^{0}}\left(Y, Y^{0}, G\right)$. We need to show that $f^{*} u \in \mathcal{A}_{\text {phg }}^{\mathcal{E}}\left(X, X^{0}, f^{*} G\right)$.

Firsi of all, note that the restriction of map $f$ to $f^{-1}\left(Y \backslash Y^{0}\right)$ determines the map $f$ : $f^{-1}\left(Y \backslash Y^{0}\right) \rightarrow Y \backslash Y^{0}$. Since $u$ is a smooth section on $Y \backslash Y^{0}, f^{*} u$ is smooth on $f^{-1}\left(Y \backslash Y^{0}\right)$, in particular, on $X \backslash X^{0}$, since $f^{-1}\left(Y^{0}\right) \subset X^{0}$.

It remains to prove that the section $f^{*} u$ is conormal at an arbitrary point $p \in X^{0}$. To be specific we suppose that $p \in X_{1} \cap \ldots \cap X_{\ell}$ and $p \notin X_{\ell+1} \cup \ldots \cup X_{r}$. Let $f(p)=p_{0}$. Suppose that $p_{0} \in Y_{1} \cap \ldots \cap Y_{\ell_{0}}$ and $p_{0} \notin Y_{\ell_{0}+1} \cup \ldots \cup Y_{r_{0}}$. We choose an adapted at $p$ coordinate system with coordinates $\left(x_{1}, \ldots, x_{\ell}, x^{0}\right) \in D_{1} \times D_{2}$ and an adapted at $p_{0}$ coordinate system with coordinates $\left(y_{1}, \ldots, y_{\ell_{0}}, y^{0}\right) \in D_{1}^{0} \times D_{2}^{0}$, where $D_{1} \subset \mathbb{R}^{\ell} ; D_{2} \subset \mathbb{R}^{m-\ell} ; D_{1}^{0} \subset \mathbb{R}^{\ell_{0}} ; D_{2}^{0} \subset \mathbb{R}^{n-\ell_{0}}$. Without loss of generality, we can assume that the restriction of bundle $G$ to the given neighborhood of $p_{0}$ is trivial, hence, we can identify the restriction of $u$ to this neighborhood with a function. Therefore, in what follows, we shall regard $u$ as a scalar function.

The case $\ell_{0}=\ell=0$ has been already treated in the beginning of the proof. In this case $p_{0} \in Y \backslash Y^{0}$ and $p \in X \backslash X^{0}$.

Consider the case $\ell_{0}=0$ and $\ell>0$. In this case $p_{0} \in Y \backslash Y^{0}$ and $p \in X^{0}$. Since $p_{0} \in Y \backslash Y^{0}$, the identities

$$
e_{f}\left(X_{j}, Y_{i}\right)=0 ; \quad \forall i=1, \ldots, r_{0} ; \quad \forall j=1, \ldots, \ell,
$$

hold true. Since $f^{*} u \in C^{\infty}\left(f^{-1}\left(Y \backslash Y^{0}\right), f^{*} G\right), f^{*} u$ is smooth at $p$, therefore, $f^{*} u$ is conormal at $p$ with respect to the trivial index family. Due to (14) it agrees with formula (5).

The further proof is given by the induction on $\ell_{0} \geqslant 1$. Since $f^{-1}\left(Y^{0}\right) \subset X^{0}, \ell>0$.

Basis of induction: $\ell_{0}=1$. In this case we have:

$$
\begin{aligned}
& e_{f}\left(X_{j}, Y_{1}\right)=0 ; \quad \forall j=\ell+1, \ldots, r \\
& e_{f}\left(X_{j}, Y_{i}\right)=0 ; \quad \forall i=2, \ldots, r_{0} ; \quad \forall j=1, \ldots, r .
\end{aligned}
$$

Since $u$ is conormal at $p_{0}$ with respect to index family $\mathcal{E}^{0}$, an expansion

$$
u\left(y_{1}, y^{0}\right) \sim \sum_{(z, q) \in E_{1}^{0}} a_{z, q}\left(y^{0}\right) y_{1}^{z} \ln ^{q}\left|y_{1}\right|,
$$

holds true, where $a_{z, q} \in C^{\infty}\left(D_{2}^{0}\right), E_{1}^{0}=\mathcal{E}^{0}\left(Y_{1}\right)$.

Since $f$ is a relative map, map $f$ is written in local coordinates as

$$
f: D_{1} \times D_{2} \subset \mathbb{R}^{\ell} \times \mathbb{R}^{n-\ell} \rightarrow D_{1}^{0} \times D_{2}^{0} \subset \mathbb{R} \times \mathbb{R}^{m-1}, \quad f:\left(x, x^{0}\right) \mapsto\left(y_{1}, y^{0}\right),
$$


where

$$
y_{1}=b_{1}\left(x, x^{0}\right) \prod_{j=1}^{\ell} x_{j}^{\gamma_{1 j}}, \quad y^{0}=g\left(x, x^{0}\right),
$$

$b_{1}$ is a smooth non-vanishing function on $D_{1} \times D_{2}$ and $g: D_{1} \times D_{2} \rightarrow D_{2}^{0}$ is a smooth map.

Let $N$ be a natural number to be chosen later. We denote $u=u_{N}+r_{N}$, where:

$$
u_{N}\left(y_{1}, y^{0}\right)=\sum_{\substack{(z, q) \in E_{1}^{0} \\ z \leqslant N}} a_{z, q}\left(y^{0}\right) y_{1}^{z} \ln ^{q}\left|y_{1}\right|
$$

Thus, we obtain $f^{*} u=f^{*} u_{N}+f^{*} r_{N}$. We have:

$$
\begin{aligned}
f^{*} u_{N}\left(x_{1}, \ldots, x_{\ell}, x^{0}\right)= & \sum_{\substack{(z, q) \in E_{1}^{0} \\
z \leqslant N}}\left(g^{*} a_{z, q}\right)\left(x, x^{0}\right) b_{1}^{z}\left(x, x^{0}\right) x_{1}^{\gamma_{11} z} \ldots x_{\ell}^{\gamma_{1 \ell} z} \\
& \quad \times\left(\ln \left|b_{1}\left(x, x^{0}\right)\right|+\gamma_{11} \ln \left|x_{1}\right|+\ldots+\gamma_{1 \ell} \ln \left|x_{\ell}\right|\right)^{q} .
\end{aligned}
$$

Since $g: D_{1} \times D_{2} \rightarrow D_{2}^{0}$ is a smooth map and $a_{z, q} \in C^{\infty}\left(D_{2}^{0}\right)$, we have $g^{*} a_{z, q} \in C^{\infty}\left(D_{1} \times D_{2}\right)$. Therefore, $f^{*} u_{N}$ can be written as

$$
f^{*} u_{N}\left(x_{1}, \ldots, x_{\ell}, x^{0}\right)=\sum_{\substack{(z, q) \in E_{1}^{0} \\ z \leqslant N}} d_{z, q}\left(x, x^{0}\right) \prod_{j=1}^{\ell} x_{j}^{\gamma_{1 j} z} \ln ^{q}\left|x_{j}\right|,
$$

where $d_{z, q} \in C^{\infty}\left(D_{1} \times D_{2}\right)$. It implies immediately that $f^{*} u_{N}$ is conormal with respect to index family $\mathcal{E}$ given by $(5)$.

By assumption, for each $\alpha_{0} \in \mathbb{Z}_{+}, \beta_{0} \in \mathbb{Z}_{+}^{m-1}$, there exists a constant $C_{1}$ such that

$$
\left|\left(y_{1} \partial_{y_{1}}\right)^{\alpha_{0}} \partial_{y^{0}}^{\beta_{0}} r_{N}\left(y_{1}, y^{0}\right)\right| \leqslant C_{1}\left|y_{1}\right|^{N+1} \text {. }
$$

By representation 16 it yields that for each $\alpha \in \mathbb{Z}_{+}^{\ell}$ and $\beta \in \mathbb{Z}_{+}^{n-\ell}$ there exists a constant $C_{3}$ such that

$$
\left|\left(x \partial_{x}\right)^{\alpha} \partial_{x^{0}}^{\beta} f^{*} r_{N}\right| \leqslant C_{3}\left|x_{1}\right|^{\gamma_{11}(N+1)}
$$

Let $N_{1}$ be an arbitrary natural number. Since $f^{*} u_{N}$ is conormal at $p$ with respect to $\mathcal{E}$, we have the representation

$$
f^{*} u_{N}\left(x_{1}, \ldots, x_{\ell}, x^{0}\right)=\sum_{\substack{(z, q) \in E_{1} \\ z \leqslant N_{1}}} h_{z, q}^{N}\left(x_{2}, \ldots, x_{\ell}, x^{0}\right) x_{1}^{z} \ln ^{q}\left|x_{1}\right|+\varrho_{N, N_{1}},
$$

where $h_{z, q}^{N}$ are conormal functions with respect to $\mathcal{E}^{\prime}=\left(\mathcal{E}\left(X_{2}\right), \ldots, \mathcal{E}\left(X_{r}\right)\right)$ and $\varrho_{N, N_{1}}$ satisfies the estimates

$$
\left|\left(x \partial_{x}\right)^{\alpha} \partial_{x^{0}}^{\beta} \varrho_{N, N_{1}}\right| \leqslant C_{6}\left|x_{2}\right|^{M_{2}} \ldots\left|x_{\ell}\right|^{M_{\ell}}\left|x_{1}\right|^{N_{1}+1}
$$

Given $N_{1}$, we choose $N$ so that the inequality

$$
N_{1}+1<\gamma_{11}(N+1)
$$

holds true. By (17), (18), (19) we have

$$
\left|\left(x \partial_{x}\right)^{\alpha} \partial_{x^{0}}^{\beta}\left(f^{*} r_{N}+\varrho_{N, N_{1}}\right)\right| \leqslant C_{7}\left|x_{2}\right|^{M_{2}^{0}} \ldots\left|x_{\ell}\right|^{M_{\ell}^{0}}\left|x_{1}\right|^{N_{1}+1},
$$

where $M_{j}^{0}=\min \left(0, M_{j}\right) \quad \forall j=2, \ldots, \ell$. Finally, we obtain that

$$
f^{*} u=\sum_{\substack{(z, q) \in E_{1} \\ z \leqslant N_{1}}} h_{z, q}^{N}\left(x_{2}, \ldots, x_{\ell}, x^{0}\right) x_{1}^{z} \ln ^{q}\left|x_{1}\right|+f^{*} r_{N}+\varrho_{N, N_{1}}
$$


It implies that $h_{z, q}^{N}$ is independent of $N$ for $N_{1}+1<\gamma_{11}(N+1)$. Denote $h_{z, q}^{N}\left(x_{2}, \ldots, x_{\ell}, x^{0}\right)=$ $h_{z, q}\left(x_{2}, \ldots, x_{\ell}, x^{0}\right)$. Hence,

$$
f^{*} u \sim \sum_{(z, q) \in E_{1}} h_{z, q}\left(x_{2}, \ldots, x_{\ell}, x^{0}\right) x_{1}^{z} \ln ^{q}\left|x_{1}\right|,
$$

and, therefore, $f^{*} u$ is a conormal function with respect to $\mathcal{E}$.

Step of induction. Fix $\ell>1$. We assume that the following statement is true. Let $Z$ and $W$ be smooth manifolds, $Z^{0}$ and $W^{0}$ be stratified submanifolds of $Z$ and $W$, respectively. We suppose that we are given a relative map $h:\left(Z, Z^{0}\right) \rightarrow\left(W, W^{0}\right)$, an arbitrary vector bundle $H$ on $W$, and an index family $\mathcal{F}^{0}$ on $W^{0}$ is given. We assume also that $p \in Z_{1} \cap \ldots \cap Z_{\ell}$ and $p \notin Z_{\ell+1} \cup \ldots \cup Z_{r}$. Let $h(p)=p_{0}$. Let $p_{0} \in W_{1} \cap \ldots \cap W_{k_{0}}$ and $p_{0} \notin W_{k_{0}+1} \cup \ldots \cup W_{r_{0}}$, and moreover $k_{0}<\ell_{0}$. We suppose that $u$ is conormal at $p_{0}$ with respect to index family $\mathcal{F}^{0}$, then $h^{*} u$ is conormal at $p$ with respect to an index family $\mathcal{F}$, where each index set $\mathcal{F}\left(Z_{j}\right)$ of index family $\mathcal{F}$ on $Z^{0}$ reads as

$$
\mathcal{F}\left(Z_{j}\right)=\left\{\left(\eta+\sum_{i} e_{h}\left(Z_{j}, W_{i}\right) z_{i}, \sum_{i} q_{i}\right) \mid\left(z_{i}, q_{i}\right) \in \mathcal{F}^{0}\left(W_{i}\right), \eta \in \mathbb{Z}_{+}\right\},
$$

where the sum is taken over all $i=1, \ldots, r_{0}$ such that $e_{f}\left(Z_{j}, W_{i}\right) \neq 0$.

Suppose that a function $u$, a map $f$, points $p$ and $p_{0}$ are as in the formulation of the theorem. Let us prove that $f^{*} u$ is a conormal function at $p$. By the assumption we have

$$
\begin{array}{lll}
e_{f}\left(X_{j}, Y_{i}\right)=0 ; & \forall i=1, \ldots, \ell_{0} ; & \forall j=\ell+1, \ldots, r \\
e_{f}\left(X_{j}, Y_{i}\right)=0 ; & \forall i=\ell_{0}+1, \ldots, r_{0} ; & \forall j=1, \ldots, r .
\end{array}
$$

Since $u$ is conormal at $p_{0}$ with respect to $\mathcal{E}^{0}$, there exists a neighborhood $V$ of $p_{0}, \varkappa_{0}(V)=$ $(-\varepsilon, \varepsilon)^{\ell_{0}} \times V_{2}$, where $V_{2} \subset \mathbb{R}^{m-\ell_{0}}$, such that $u$ is defined and smooth on $V \backslash X^{0}$, and, for each $\left(y_{2}, \ldots, y_{\ell_{0}}, y^{0}\right) \in(-\varepsilon, \varepsilon)^{\ell_{0}-1} \times V_{2}$, the asymptotic expansion

$$
u\left(y, y^{0}\right) \sim \sum_{(z, q) \in E_{1}^{0}} a_{z, q}\left(y_{2}, \ldots, y_{\ell_{0}}, y^{0}\right) y_{1}^{z} \ln ^{q}\left|y_{1}\right|
$$

is valid as $y_{1} \rightarrow 0$, where $E_{1}^{0}=\mathcal{E}^{0}\left(Y_{1}\right)$, functions $a_{z, q}$ are conormal on $(-\varepsilon, \varepsilon)^{\ell_{0}-1} \times V_{2} \subset Z$ with respect to $\mathcal{E}_{0}^{\prime}$.

Here we consider $Z=\mathbb{R}^{\ell_{0}-1} \times \mathbb{R}^{m-\ell_{0}}$ with coordinates $\left(y_{2}, \ldots, y_{\ell_{0}}, y^{0}\right)$, where $y_{j} \in \mathbb{R}, j=$ $2, \ldots, \ell_{0}, y^{0} \in \mathbb{R}^{m-\ell_{0}}$, equipped with a stratified submanifold $Z^{0}=\left\{y_{2}=0\right\} \cup \ldots \cup\left\{y_{\ell_{0}}=0\right\}$. An index family $\mathcal{E}_{0}^{\prime}$ on $Z^{0}$ is introduced as $\mathcal{E}_{0}^{\prime}\left(\left\{y_{j}=0\right\}\right)=E_{j}^{0}$, where $j=2, \ldots, \ell_{0}$.

Since $f$ is a relative map, in local coordinates the map $f$ is written as

$$
f: D_{1} \times D_{2} \subset \mathbb{R}^{\ell} \times \mathbb{R}^{n-\ell} \rightarrow \mathbb{R}^{\ell_{0}} \times \mathbb{R}^{m-\ell_{0}}, \quad\left(x, x^{0}\right) \mapsto\left(y_{1}, \ldots, y_{\ell_{0}}, y^{0}\right),
$$

where

$$
y_{i}=b_{i}\left(x, x^{0}\right) \prod_{j=1}^{\ell} x_{j}^{\gamma_{i j}}, \quad i=1, \ldots, \ell_{0}, \quad y^{0}=F\left(x, x^{0}\right),
$$

$b_{i}$ are smooth non-vanishing functions on $X$.

We introduce the map

$$
g: D_{1} \times D_{2} \subset \mathbb{R}^{\ell} \times \mathbb{R}^{n-\ell} \rightarrow \mathbb{R}^{\ell_{0}-1} \times \mathbb{R}^{m-\ell_{0}}, \quad\left(x, x^{0}\right) \mapsto\left(y_{2}, \ldots, y_{\ell_{0}}, y^{0}\right),
$$

where

$$
y_{i}=b_{i}\left(x, x^{0}\right) \prod_{j=1}^{\ell} x_{j}^{\gamma_{i j}}, \quad i=2, \ldots, \ell_{0}, \quad y^{0}=F\left(x, x^{0}\right) .
$$

We observe that $g$ is a relative map, and moreover,

$$
e_{g}\left(X_{j}, Y_{i}\right)=e_{f}\left(X_{j}, Y_{i}\right) ; \quad \forall j=1, \ldots, \ell ; \quad \forall i=2, \ldots, \ell_{0} .
$$


Let $N$ be a natural number to be chosen later. We denote $u=u_{N}+r_{N}$, where

$$
u_{N}\left(y, y^{0}\right)=\sum_{\substack{(z, q) \in E_{1}^{0} \\ z \leqslant N}} a_{z, q}\left(y_{2}, \ldots, y_{\ell_{0}}, y^{0}\right) y_{1}^{z} \ln ^{q}\left|y_{1}\right|
$$

Hence, we obtain $f^{*} u=f^{*} u_{N}+f^{*} r_{N}$. We have

$$
\begin{aligned}
f^{*} u_{N}\left(x_{1}, \ldots, x_{\ell}, x^{0}\right)= & \sum_{\substack{(z, q) \in E_{1}^{0} \\
z \leqslant N}}\left(g^{*} a_{z, q}\right)\left(x, x^{0}\right) b_{1}^{z}\left(x, x^{0}\right) x_{1}^{\gamma_{11} z} \ldots x_{\ell}^{\gamma_{1 \ell} z} \\
& \quad \times\left(\ln \left|b_{1}\left(x, x^{0}\right)\right|+\gamma_{11} \ln \left|x_{1}\right|+\ldots+\gamma_{1 \ell} \ln \left|x_{\ell}\right|\right)^{q} .
\end{aligned}
$$

There exists a neighborhood $U$ of $p, \varkappa(U)=(-\delta, \delta)^{\ell} \times U_{2}$, where $U_{2} \subset \mathbb{R}^{n-\ell}$, such that $g(U) \subset V$. Since $g$ is a relative map, $a_{z, q} \in \mathcal{A}_{\text {phg }}^{\mathcal{E}^{\prime}}\left((-\varepsilon, \varepsilon)^{\ell_{0}-1} \times V_{2}\right)$, by (21) and the induction hypothesis, we obtain that $g^{*} a_{z, q} \in \mathcal{A}_{p h g}^{\tilde{\mathcal{E}}}\left((-\delta, \delta)^{\ell} \times U_{2}\right)$, where index set $\tilde{\mathcal{E}}\left(X_{j}\right)$ of index family $\tilde{\mathcal{E}}$ reads as

$$
\tilde{\mathcal{E}}\left(X_{j}\right)=\left\{\left(\eta+\sum_{i=2}^{r_{0}} e_{f}\left(X_{j}, Y_{i}\right) z_{i}, \sum_{i=2}^{r_{0}} q_{i}\right) \mid\left(z_{i}, q_{i}\right) \in \mathcal{E}^{0}\left(Y_{i}\right), \eta \in \mathbb{Z}_{+}\right\},
$$

where the sum is taken over all $i=2, \ldots, r_{0}$ such that $e_{f}\left(X_{j}, Y_{i}\right) \neq 0$.

Hence, $f^{*} u_{N}$ can be written as

$$
f^{*} u_{N}\left(x_{1}, \ldots, x_{\ell}, x^{0}\right)=\sum_{\substack{(z, q) \in E_{1}^{0} \\ z \leqslant N}} d_{z, q}\left(x, x^{0}\right) \prod_{j=1}^{\ell} x_{j}^{\gamma_{1 j} z} \ln ^{q}\left|x_{j}\right|,
$$

where $d_{z, q} \in \mathcal{A}_{\text {phg }}^{\tilde{\mathcal{E}}}\left((-\delta, \delta)^{\ell} \times U_{2}\right)$. It implies that $f^{*} u_{N}$ is conormal with respect to $\mathcal{E}$.

By assumption, there exist real numbers $M_{2}, \ldots, M_{\ell_{0}}$ such that for each $\alpha \in \mathbb{Z}_{+}^{\ell_{0}}$ and $\beta \in$ $\mathbb{Z}_{+}^{m-\ell_{0}}$ there exists a constant $C=C_{\alpha \beta N}$ such that

$$
\left|\left(y \partial_{y}\right)^{\alpha} \partial_{y^{0}}^{\beta} r_{N}\left(y, y^{0}\right)\right| \leqslant C\left|y_{2}\right|^{M_{2}} \cdot \ldots \cdot\left|y_{\ell_{0}}\right|^{M_{\ell_{0}}}\left|y_{1}\right|^{N+1} .
$$

It yields that for $\left|x_{j}\right|<1$

$$
\begin{aligned}
\left|f^{*} r_{N}\left(x, x^{0}\right)\right| & \leqslant C_{1}\left|x_{2}\right|^{M_{2}^{0}+\gamma_{12}(N+1)} \ldots\left|x_{\ell}\right|^{M_{\ell}^{0}+\gamma_{1 \ell}(N+1)}\left|x_{1}\right|^{\gamma_{11}(N+1)+M_{1}^{0}} \\
& \leqslant C_{4}\left|x_{2}\right|^{M_{2}^{0}} \ldots\left|x_{\ell}\right|^{M_{\ell}^{0}}\left|x_{1}\right|^{\gamma_{11}(N+1)+M_{1}^{0}}
\end{aligned}
$$

where

$$
M_{j}^{0}=\sum_{i=2}^{\ell_{0}} \gamma_{i j} M_{i}, \quad j=1, \ldots, \ell
$$

Similar estimates hold for derivatives

$$
\left|\left(x \partial_{x}\right)^{\alpha} \partial_{x^{0}}^{\beta} f^{*} r_{N}\right| \leqslant C_{5}\left|x_{2}\right|^{M_{2}^{0}} \ldots\left|x_{\ell}\right|^{M_{\ell}^{0}}\left|x_{1}\right|^{\gamma_{11}(N+1)+M_{1}^{0}} .
$$

As in the case $\ell=1$, by the above relations one can conclude that $f^{*} u$ is a conormal function with respect to index set $\mathcal{E}$.

\section{B. Proof of Theorem 6}

Let $\mu \in \mathcal{A}_{\text {phg }}^{\mathcal{E}}\left(X, X^{0}, f^{*} G \otimes \Omega_{X}\right)$. Let us show that $f_{*} \mu$ is well-defined and $f_{*} \mu \in$ $\mathcal{A}_{\text {phg }}^{\mathcal{E}^{0}}\left(Y, Y^{0}, G \otimes \Omega_{Y}\right)$.

Let $p_{0} \notin Y^{0}$. Let us show that $f_{*} \mu$ is a smooth density at $p_{0}$. We choose a local coordinate system with coordinates $y^{0} \in D_{2}^{0} \subset \mathbb{R}^{m}$ in a neighborhood of $p_{0}$ and take an arbitrary point $p \in X$ such that $f(p)=p_{0}$. We suppose that $p \in X_{1} \cap \ldots \cap X_{\ell}$ and $p \notin X_{\ell+1} \cup \ldots \cup X_{r}$. We choose 
an adapted at $p$ coordinate system with coordinates $\left(x, x^{0}\right) \in D_{1} \times D_{2} \subset \mathbb{R}^{\ell} \times \mathbb{R}^{n-\ell}$. Since $f$ is a relative fibration, in local coordinates map $f$ reads as $y^{0}=f\left(x, x^{0}\right)$, where rank $\left(\frac{\partial f}{\partial x^{0}}\right)=m$. Hence, one can choose an adapted at $p$ coordinate system such that $f$ becomes a projection:

$$
y^{0}=f\left(x, x^{0}\right)=\left(x_{1}^{0}, \ldots, x_{m}^{0}\right), \quad x \in D_{1}, \quad x^{0} \in D_{2} .
$$

By compactness of $X$, there exists a finite family of neighborhoods $V_{p_{s}}, s=1, \ldots, d$, such that $X=\left(X \backslash f^{-1}\left(p_{0}\right)\right) \bigcup \bigcup_{s=1}^{d} V_{p_{s}}$. Let $\psi_{s} \in C^{\infty}(X), s=0, \ldots, d$, be a smooth partition of unity subordinated to this covering: $\operatorname{supp} \psi_{0} \subset X \backslash f^{-1}\left(p_{0}\right), \operatorname{supp} \psi_{s} \subset V_{p_{s}}$ for $s=1, \ldots, d$, $\psi_{s} \geqslant 0, \sum_{s=0}^{d} \psi_{s}=1$. There exists a neighborhood $U_{p_{0}}$ of $p_{0}$ such that $\sum_{s=1}^{d} \psi_{s}(m)=1$ for each $m \in f^{-1}\left(U_{p_{0}}\right)$.

As in the proof of Theorem 5, without loss of generality one can assume that bundle $G$ is trivial and $\mu$ is a density on $X$. In coordinate neighborhood $V_{p_{s}}$, density $\mu$ is written as

$$
\mu=\mu\left(x, x^{0}\right)\left|\frac{d x}{x} d x^{0}\right| .
$$

We choose $\varphi \in C_{0}^{\infty}(Y)$ such that $\operatorname{supp} \varphi \subset U_{p_{0}}$. Then $f^{*} \varphi \in C^{\infty}(X)$, moreover,

$$
\left\langle f_{*} \mu, \varphi\right\rangle=\left\langle\mu, f^{*} \varphi\right\rangle=\int_{f^{-1}\left(U_{p_{0}}\right)} \mu(m) \varphi(f(m)) .
$$

Using the partition of unity and the local coordinates, we obtain

$$
\left\langle f_{*} \mu, \varphi\right\rangle=\sum_{s=1}^{d} \int_{D_{1} \times D_{2}} \psi_{s}\left(x, x^{0}\right) \mu\left(x, x^{0}\right) \varphi\left(f\left(x, x^{0}\right)\right) \frac{d x}{x} d x^{0} .
$$

Taking into consideration formula $(23)$, the latter identity can re-written as

$$
\left\langle f_{*} \mu, \varphi\right\rangle=\int_{U_{p_{0}}} F\left(y^{0}\right) \varphi\left(y^{0}\right) d y^{0}
$$

where $F$ is given by

$$
\begin{aligned}
F\left(y^{0}\right)=\sum_{s=1}^{d} \int_{\mathbb{R}^{\ell} \times \mathbb{R}^{n-\ell-m}} & \psi_{s}\left(x, y^{0}, x_{m+1}^{0}, \ldots, x_{n-\ell}^{0}\right) \\
& \times \mu\left(x, y^{0}, x_{m+1}^{0}, \ldots, x_{n-\ell}^{0}\right) \frac{d x}{x} d x_{m+1}^{0} \ldots d x_{n-\ell}^{0} .
\end{aligned}
$$

Since $p \in X_{1} \cap \ldots \cap X_{\ell}, p \notin X_{\ell+1} \cup \ldots \cup X_{r}$, and $f(p) \notin Y^{0}$, we have $e_{f}\left(X_{j}, Y_{i}\right)=0$ if $j=1, \ldots, \ell$, $i=1, \ldots, r_{0}$. It yields that $\inf \mathcal{E}\left(X_{j}\right)>0$ for each $j=1, \ldots, \ell$. Hence, the estimate

$$
\left|\mu\left(x, y^{0}, x_{m+1}^{0}, \ldots, x_{n-\ell}^{0}\right)\right|<C\left|x_{1}\right|^{\varepsilon_{1}} \cdot \ldots \cdot\left|x_{\ell}\right|^{\varepsilon_{\ell}}
$$

holds true, where $\varepsilon_{1}, \ldots, \varepsilon_{\ell}$ are positive numbers. It implies that the integral in the right-hand side of (26) converges uniformly, and therefore, function $F$ is smooth in a neighborhood of $p_{0}$. According to (25), the restriction of density $f_{*} \mu$ to $U_{p_{0}}$ is well-defined and coincides with the smooth density $F\left(y^{0}\right)\left|d y^{0}\right|$. Therefore, $f_{*} \mu$ is well-defined as a smooth density on $Y \backslash Y^{0}$.

Let $p_{0} \in Y^{0}$ and suppose that $p_{0} \in Y_{1} \cup \ldots \cup Y_{\ell_{0}}$ and $p_{0} \notin Y_{\ell_{0}+1} \cup \ldots \cup Y_{r_{0}}, \ell_{0} \neq 0$. Let us prove that $f_{*} \mu$ is conormal at $p_{0}$.

The case $\ell_{0}=1$. We choose an adapted at $p_{0}$ coordinate system with coordinates $\left(y_{1}, y^{0}\right) \in$ $D_{1}^{0} \times D_{2}^{0} \subset \mathbb{R} \times \mathbb{R}^{m-1}$ and $p \in X$ such that $f(p)=p_{0}$. We assume that $p \in X_{1} \cap \ldots \cap X_{\ell}$ and $p \notin X_{\ell+1} \cup \ldots \cup X_{r}$. We choose an adapted at $p$ coordinate system with coordinates $\left(x, x^{0}\right) \in$ 
$D_{1} \times D_{2} \subset \mathbb{R}^{\ell} \times \mathbb{R}^{n-\ell}$. In these coordinate systems, map $f$ is written as $\left(y_{1}, y^{0}\right)=f\left(x, x^{0}\right)$, where $y_{1}=b_{1}\left(x, x^{0}\right) x_{1}^{\gamma_{11}} \ldots x_{\ell}^{\gamma_{1 \ell}}$, function $b_{1}$ is smooth and non-vanishing; $y^{0}=g\left(x, x^{0}\right)$. Since $f(p)=p_{0}$, at least one of $\gamma_{11}, \gamma_{12}, \ldots, \gamma_{1 \ell}$ is positive. To be specific let $\gamma_{11}>0$. Then, without loss of generality one can assume that $b_{1}\left(x, x^{0}\right) \equiv 1$, because one can make a change of variables in a neighborhood of zero:

$$
\widetilde{x}_{1}=b_{1}\left(x, x^{0}\right)^{\frac{1}{\gamma_{11}}} x_{1} ; \quad \widetilde{x}_{j}=x_{j}, \quad \forall j=2, \ldots, \ell ; \quad \widetilde{x}^{0}=x^{0} .
$$

The Jacobian of this change will be denoted by $w\left(x, x^{0}\right)$. It is easy to see that $w\left(0, x^{0}\right) \neq 0$ for each $x^{0} \in D_{2}$.

By Condition (4) of Definition 13 we have rank $\left(\frac{\partial g}{\partial x^{0}}\right)=m-1$. Hence, one can choose an adapted at $p$ coordinate system such that $g$ becomes a projection:

$$
y^{0}=g\left(x, x^{0}\right)=\left(x_{1}^{0}, \ldots, x_{m-1}^{0}\right), \quad x \in D_{1}, \quad x^{0} \in D_{2} .
$$

By compactness of $X$, there exists a finite family of neighborhoods $V_{p_{s}}, s=1, \ldots, d$, such that $X=\left(X \backslash f^{-1}\left(p_{0}\right)\right) \cup \bigcup_{s=1}^{d} V_{p_{s}}$. Let $\psi_{s} \in C^{\infty}(X), s=0, \ldots, d$ be a smooth partition of unity subordinated to this covering: $\operatorname{supp} \psi_{0} \subset X \backslash f^{-1}\left(p_{0}\right), \operatorname{supp} \psi_{s} \subset V_{p_{s}}$ for $s=1, \ldots, d$, $\psi_{s} \geqslant 0, \sum_{s=0}^{d} \psi_{s}=1$. There exists a neighborhood $U_{p_{0}}$ of $p_{0}$ such that $\sum_{s=1}^{d} \psi_{s}(m)=1$ for any $m \in f^{-1}\left(U_{p_{0}}\right)$.

As above, we will assume that bundle $G$ is trivial and $\mu$ is a density on $X$. In coordinate neighborhood $V_{p_{s}}$, density $\mu$ is written as

$$
\mu=\mu\left(x, x^{0}\right)\left|\frac{d x}{x} d x^{0}\right| .
$$

We choose $\varphi \in C_{0}^{\infty}(Y)$ such that $\operatorname{supp} \varphi \subset U_{p_{0}}$. Then $f^{*} \varphi \in C^{\infty}(X)$, moreover,

$$
\left\langle f_{*} \mu, \varphi\right\rangle=\left\langle\mu, f^{*} \varphi\right\rangle=\int_{f^{-1}\left(U_{p_{0}}\right)} \mu(m) \varphi(f(m)) .
$$

Employing the partition of unity and the local coordinates, we obtain that

$$
\left\langle f_{*} \mu, \varphi\right\rangle=\sum_{s=1}^{d} \int_{\mathbb{R}^{\ell} \times \mathbb{R}^{n-\ell}} \psi_{s}\left(x, x^{0}\right) \mu\left(x, x^{0}\right) \varphi\left(x_{1}^{\gamma_{11}} \ldots x_{\ell}^{\gamma_{1 \ell}}, x_{1}^{0}, \ldots, x_{m-1}^{0}\right) \frac{d x}{x} d x^{0} .
$$

Since $\ell_{0}=1$, by Definition 11 at least one of $\gamma_{11}, \gamma_{12}, \ldots, \gamma_{1 \ell}$ is positive. To be specific let $\gamma_{11}, \gamma_{12}, \ldots, \gamma_{1 k_{1}}>0, \gamma_{1, k_{1}+1}=\ldots=\gamma_{1 \ell}=0$, where $k_{1} \leqslant \ell$. Denote $\mu_{s}\left(x, x^{0}\right)=$ $\frac{1}{\gamma_{11}} \psi_{s}\left(x, x^{0}\right) \mu\left(x, x^{0}\right)$. Identity (27) casts into the form

$$
\left\langle f_{*} \mu, \varphi\right\rangle=\gamma_{11} \sum_{s=1}^{d} \int_{\mathbb{R}^{\ell} \times \mathbb{R}^{n-\ell}} \mu_{s}\left(x, x^{0}\right) \varphi\left(x_{1}^{\gamma_{11}} x_{2}^{\gamma_{12}} \ldots x_{k_{1}}^{\gamma_{1 k_{1}}}, x_{1}^{0}, \ldots, x_{m-1}^{0}\right) \frac{d x}{x} d x^{0} .
$$

Making the change of variables

$$
y_{1}=x_{1}^{\gamma_{11}} \ldots x_{k_{1}}^{\gamma_{1 k_{1}}}, \quad t_{j}=x_{j} \quad \forall j=2, \ldots \ell ; \quad y^{0}=\left(x_{1}^{0}, \ldots, x_{m-1}^{0}\right)
$$

in the integral, we obtain that

$$
\begin{gathered}
\left\langle f_{*} \mu, \varphi\right\rangle=\sum_{s=1}^{d} \int_{\mathbb{R}^{\ell} \times \mathbb{R}^{n-\ell}} \mu_{s}\left(y_{1}^{\frac{1}{\gamma_{11}}} t_{2}^{\frac{-\gamma_{12}}{\gamma_{11}}} \ldots t_{k_{1}}^{-\frac{\gamma_{1 k_{1}}}{\gamma_{11}}}, t_{2}, \ldots, t_{\ell}, y^{0}, x_{m}^{0}, \ldots, x_{n-\ell}^{0}\right) \\
\times \varphi\left(y_{1}, y^{0}\right) \frac{d y_{1}}{y_{1}} \frac{d t_{2}}{t_{2}} \ldots \frac{d t_{\ell}}{t_{\ell}} d y^{0} d x_{m}^{0} \ldots d x_{n-\ell}^{0} .
\end{gathered}
$$


Hence, for each $\left(y_{1}, y^{0}\right)$ in some neighborhood of $p_{0}$, density $f_{*} \mu$ is given by

$$
f_{*} \mu=\sum_{s=1}^{d} \nu_{s}\left(y_{1}, y^{0}\right)\left|\frac{d y_{1}}{y_{1}} d y^{0}\right|
$$

where functions $\nu_{s}\left(y_{1}, y^{0}\right)$ read as

$$
\begin{aligned}
& \nu_{s}\left(y_{1}, y^{0}\right)= \int_{\mathbb{R}^{\ell-1} \times \mathbb{R}^{n-m-\ell+1}} \mu_{s}\left(y_{1}^{\frac{1}{\gamma_{11}}} t_{2}^{-\frac{\gamma_{12}}{\gamma_{11}}} \ldots t_{k_{1}}^{-\frac{\gamma_{1 k_{1}}}{\gamma_{11}}}, t_{2}, \ldots, t_{\ell}, y^{0}, x_{m}^{0}, \ldots, x_{n-\ell}^{0}\right) \\
& \frac{d t_{2}}{t_{2}} \ldots \frac{d t_{\ell}}{t_{\ell}} d x_{m}^{0} \ldots d x_{n-\ell}^{0}
\end{aligned}
$$

Since for $j=k_{1}+1, \ldots, \ell$ we have inf $E_{j}>0$, the integral in the last formula converges, therefore, $\nu_{s}$ is a smooth function for $y_{1} \neq 0$.

Fix $s$. Let us prove that function $\nu_{s}$ is conormal at $y_{1}=0$ with respect to index set $E_{1}^{0}$. We write

$$
\nu_{s}\left(y_{1}, y^{0}\right)=\int_{\mathbb{R}^{\ell-k_{1}}} \mu_{s}^{1}\left(y_{1}, t_{k_{1}+1}, \ldots, t_{\ell}, y^{0}\right) \frac{d t_{k_{1}+1}}{t_{k_{1}+1}} \ldots \frac{d t_{\ell}}{t_{\ell}},
$$

where

$$
\begin{gathered}
\mu_{s}^{1}\left(y_{1}, t_{k_{1}+1}, \ldots, t_{\ell}, y^{0}\right)=\int_{\mathbb{R}^{k_{1}-1} \times \mathbb{R}^{n-m-\ell+1}} \mu_{s}\left(y_{1}^{\frac{1}{\gamma_{11}}} t_{2}^{-\frac{\gamma_{12}}{\gamma_{11}}} \ldots t_{k_{1}}^{-\frac{\gamma_{1 k_{1}}}{\gamma_{11}}}, t_{2}, \ldots, t_{\ell}, y^{0}, x_{m}^{0}, \ldots, x_{n-\ell}^{0}\right) \\
\frac{d t_{2}}{t_{2}} \ldots \frac{d t_{k_{1}}}{t_{k_{1}}} d x_{m}^{0} \ldots d x_{n-\ell}^{0} .
\end{gathered}
$$

The proof of Theorem 6 for $\ell_{0}=1$ is completed by means of the following statement.

Proposition 2. If a function $\mu_{s}\left(x_{1}, \ldots, x_{\ell}, y^{0}, x_{m}^{0}, \ldots, x_{n-\ell}^{0}\right)$ is compactly supported and conormal in variables $\left(x_{1}, \ldots, x_{\ell}\right)$ with respect to an index family $\left(E_{1}, \ldots, E_{\ell}\right)$ and $\gamma_{11}, \gamma_{12}, \ldots, \gamma_{1 k_{1}}>0$, then function $\mu_{s}^{1}$ given by formula (29) is conormal in variables $\left(y_{1}, t_{k_{1}+1}, \ldots, t_{\ell}\right)$ with respect to index family $\left(E_{1}^{0}, E_{k_{1}+1}, \ldots, E_{\ell}\right)$, where

$$
E_{1}^{0}=\bar{\bigcup}_{j=1, \ldots, k_{1}}\left\{\left(\frac{z}{\gamma_{1 j}}, q\right):(z, q) \in E_{j}\right\} .
$$

Once Proposition 2 is proved, by applying Theorem 6 to function $\mu_{s}^{1}$ in the case $\ell_{0}=0$ and taking into consideration that, for $j=k_{1}+1, \ldots, \ell$ we have $\inf E_{j}>0$, it follows from (28) that the function $\nu_{s}$ is conormal at $y_{1}=0$ that completes the proof of Theorem 6 for $\ell_{0}=1$.

Proof of Proposition 2. Since for $y_{1} \neq 0$ the integrand is a smooth compactly supported function, the integral converges absolutely, and $\mu_{s}^{1}$ is a smooth function.

Let us prove that function $\mu_{s}^{1}$ is conormal at $y_{1}=0$.

The case $k_{1}=\ell=1$. In this case, function $\mu_{s}^{1}$ reads as

$$
\mu_{s}^{1}\left(y_{1}, y^{0}\right)=\int_{\mathbb{R}^{n-m}} \mu_{s}\left(y_{1}^{\frac{1}{\gamma_{11}}}, y^{0}, x_{m}^{0}, \ldots, x_{n-1}^{0}\right) d x_{m}^{0} \ldots d x_{n-1}^{0} .
$$

Since $\mu_{s}$ is a conormal function at $x_{1}=0$ with respect to index set $E_{1}$, we have:

$$
\mu_{s}\left(x_{1}, x^{0}\right) \sim \sum_{(z, q) \in E_{1}} a_{z, q}\left(x^{0}\right) x_{1}^{z} \ln ^{q}\left|x_{1}\right|,
$$


where $a_{z, q}$ are smooth functions. Denote $\mu_{s}=\mu_{N}+r_{N}$, where

$$
\mu_{N}\left(x_{1}, x^{0}\right)=\sum_{\substack{(z, q) \in E_{1} \\ z \leqslant N}} a_{z, q}\left(x^{0}\right) x_{1}^{z} \ln ^{q}\left|x_{1}\right|,
$$

$N$ is a natural number to be chosen later. By (30), function $\mu_{s}^{1}$ is represented as $\mu_{s}^{1}=\nu_{N}+\tilde{r}_{N}$, where

$$
\nu_{N}\left(y_{1}, y^{0}\right)=\frac{1}{\gamma_{11}^{q}} \sum_{\substack{(z, q) \in E_{1} \\ z \leqslant N}} \int_{\mathbb{R}^{n-m}} a_{z, q}\left(y^{0}, x_{m}^{0}, \ldots, x_{n-1}^{0}\right) y_{1}^{\frac{z}{\gamma_{11}}} \ln ^{q}\left|y_{1}\right| d x_{m}^{0} \ldots d x_{n-1}^{0}
$$

and

$$
\tilde{r}_{N}\left(y_{1}, y^{0}\right)=\int_{\mathbb{R}^{n-m}} r_{N}\left(y_{1}^{\frac{1}{\gamma_{11}}}, y^{0}, x_{m}^{0}, \ldots, x_{n-1}^{0}\right) d x_{m}^{0} \ldots d x_{n-1}^{0} .
$$

We have

$$
\nu_{N}\left(y_{1}, y^{0}\right)=\sum_{\substack{(z, q) \in E_{1} \\ z \leqslant N}} h_{z, q}\left(y^{0}\right) y_{1}^{\frac{z}{\gamma_{11}}} \ln ^{q}\left|y_{1}\right|
$$

where

$$
h_{z, q}\left(y^{0}\right)=\frac{1}{\gamma_{11}^{q}} \int_{\mathbb{R}^{n-m}} a_{z, q}\left(y^{0}, x_{m}^{0}, \ldots, x_{n-1}^{0}\right) d x_{m}^{0} \ldots d x_{n-1}^{0} .
$$

Since $a_{z, q}$ are smooth compactly supported functions, function $\nu_{N}$ is conormal at $y_{1}=0$ with respect to the index set $E_{1}^{0}=\left\{\left(\frac{z}{\gamma_{11}}, q\right):(z, q) \in E_{1}\right\}$.

By definition, for each $\alpha_{0} \in \mathbb{Z}_{+}$and for each multi-index $\beta_{0}$, there exists a constant $C_{1}$ such that

$$
\left|\left(x_{1} \frac{\partial}{\partial x_{1}}\right)^{\alpha_{0}} \partial_{x^{0}}^{\beta_{0}} r_{N}\left(x_{1}, x^{0}\right)\right|<C_{1}\left|x_{1}\right|^{N+1} .
$$

Therefore, for each $\alpha \in \mathbb{Z}_{+}$and for each multi-index $\beta$, there exists a constant $C_{2}$ such that

$$
\left|\left(y_{1} \frac{\partial}{\partial y_{1}}\right)^{\alpha} \partial_{y^{0}}^{\beta_{0}} \tilde{r}_{N}\left(y_{1}, y^{0}\right)\right|<C_{2}\left|y_{1}\right|^{\frac{N+1}{\gamma_{11}}} \text {. }
$$

It implies immediately that

$$
\mu_{s}^{1}\left(y_{1}, y^{0}\right) \sim \sum_{(z, q) \in E_{1}^{0}} h_{z, q}\left(y^{0}\right) y_{1}^{z} \ln ^{q}\left|y_{1}\right|
$$

The case $k_{1}=\ell=2$. In this case, the function $\mu_{s}^{1}$ reads as

$$
\mu_{s}^{1}\left(y_{1}, y^{0}\right)=\int_{\mathbb{R} \times \mathbb{R}^{n-m-1}} \mu_{s}\left(y_{1}^{\frac{1}{\gamma_{11}}} t^{\frac{-\gamma_{12}}{\gamma_{11}}}, t, y^{0}, x_{m}^{0}, \ldots, x_{n-2}^{0}\right) \frac{d t}{t} d x_{m}^{0} \ldots d x_{n-2}^{0} .
$$

Since function $\mu_{s}\left(x_{1}, x_{2}, x^{0}\right)$ is conormal at $\left(x_{1}, x_{2}\right)=(0,0)$ with respect to the index family $\left(E_{1}, E_{2}\right)$, we have:

$$
\mu_{s}\left(x_{1}, x_{2}, x^{0}\right) \sim \sum_{\left(z_{1}, q_{1}\right) \in E_{1}} a_{z_{1}, q_{1}}\left(x_{2}, x^{0}\right) x_{1}^{z_{1}} \ln ^{q_{1}}\left|x_{1}\right|,
$$

where $a_{z_{1}, q_{1}}\left(x_{2}, x^{0}\right)$ are conormal functions at $x_{2}=0$ with respect to index set $E_{2}$. By definition, for each natural $N_{1}$ the representation

$$
\mu_{s}\left(x_{1}, x_{2}, x^{0}\right)=\sum_{\substack{\left(z_{1}, q_{1}\right) \in E_{1} \\ z_{1} \leqslant N_{1}}} a_{z_{1}, q_{1}}\left(x_{2}, x^{0}\right) x_{1}^{z_{1}} \ln ^{q_{1}}\left|x_{1}\right|+r_{N_{1}}\left(x_{1}, x_{2}, x^{0}\right)
$$

is valid. 
Function $a_{z_{1}, q_{1}}\left(x_{2}, x^{0}\right)$ admits the asymptotic expansion

$$
a_{z_{1}, q_{1}} \sim \sum_{\left(z_{2}, q_{2}\right) \in E_{2}} b_{z_{1}, q_{1}, z_{2}, q_{2}}\left(x^{0}\right) x_{2}^{z_{2}} \ln ^{q_{2}}\left|x_{2}\right|,
$$

$b_{z_{1}, q_{1}, z_{2}, q_{2}}$ are smooth functions. Therefore, for each natural $N_{2}$ the representation

$$
a_{z_{1}, q_{1}}\left(x_{2}, x^{0}\right)=a_{z_{1} q_{1} N_{2}}\left(x_{2}, x^{0}\right)+r_{z_{1} q_{1} N_{2}}\left(x_{2}, x^{0}\right)
$$

holds true, where

$$
a_{z_{1} q_{1} N_{2}}=\sum_{\substack{\left(z_{2}, q_{2}\right) \in E_{2} \\ z_{2} \leqslant N_{2}}} b_{z_{1}, q_{1}, z_{2}, q_{2}}\left(x^{0}\right) x_{2}^{z_{2}} \ln ^{q_{2}}\left|x_{2}\right| .
$$

Thus, we arrive at the representation

$$
\mu_{s}\left(x_{1}, x_{2}, x^{0}\right)=\mu_{N_{1} N_{2}}\left(x_{1}, x_{2}, x^{0}\right)+r_{N_{1} N_{2}}\left(x_{1}, x_{2}, x^{0}\right),
$$

where

$$
\begin{aligned}
& \mu_{N_{1} N_{2}}\left(x_{1}, x_{2}, x^{0}\right)=\sum_{\substack{\left(z_{1}, q_{1}\right) \in E_{1} \\
z_{1} \leqslant N_{1}}} \sum_{\substack{\left.z_{2}, q_{2}\right) \in E_{2} \\
z_{2} \leqslant N_{2}}} b_{z_{1}, q_{1}, z_{2}, q_{2}}\left(x^{0}\right) x_{2}^{z_{2}} x_{1}^{z_{1}} \ln ^{q_{2}}\left|x_{2}\right| \ln ^{q_{1}}\left|x_{1}\right|, \\
& r_{N_{1} N_{2}}\left(x_{1}, x_{2}, x^{0}\right)=r_{N_{1}}\left(x_{1}, x_{2}, x^{0}\right)+\sum_{\substack{\left(z_{1}, q_{1}\right) \in E_{1} \\
z_{1} \leqslant N_{1}}} r_{z_{1} q_{1} N_{2}}\left(x_{2}, x^{0}\right) x_{1}^{z_{1}} \ln ^{q_{1}}\left|x_{1}\right|,
\end{aligned}
$$

$N_{1}, N_{2}$ are natural numbers to be chosen later.

By assumption, there exists $M_{1}$ such that for each $\alpha_{1}, \alpha_{2} \in \mathbb{Z}_{+}$and for each multi-index $\beta_{2}$, there exists a constant $C_{1}$ such that

$$
\left|\left(x_{1} \partial_{x_{1}}\right)^{\alpha_{1}}\left(x_{2} \partial_{x_{2}}\right)^{\alpha_{2}} \partial_{x^{0}}^{\beta_{2}} r_{N_{1}}\left(x_{1}, x_{2}, x^{0}\right)\right|<C_{1}\left|x_{1}\right|^{M_{1}}\left|x_{2}\right|^{N_{2}+1} .
$$

Moreover, for each $\alpha_{2} \in \mathbb{Z}_{+}$and for each multi-index $\beta_{2}$, there exists a constant $C_{2}$ such that

$$
\left|\left(x_{2} \partial_{x_{2}}\right)^{\alpha_{2}} \partial_{x^{0}}^{\beta_{2}} r_{z_{1} q_{1} N_{2}}\left(x_{2}, x^{0}\right)\right|<C_{2}\left|x_{2}\right|^{N_{2}+1} .
$$

It implies that there exists $\tilde{M}_{1}$ such that for each $\alpha_{1}, \alpha_{2} \in \mathbb{Z}_{+}$and for each multi-index $\beta_{2}$, there exists a constant $C_{1}$ such that

$$
\left|\left(x_{1} \partial_{x_{1}}\right)^{\alpha_{1}}\left(x_{2} \partial_{x_{2}}\right)^{\alpha_{2}} \partial_{x^{0}}^{\beta_{2}} r_{N_{1} N_{2}}\left(x_{1}, x_{2}, x^{0}\right)\right|<C_{1}\left|x_{1}\right|^{\tilde{M}_{1}}\left|x_{2}\right|^{N_{2}+1} .
$$

Taking into consideration the fact that $\mu_{s}\left(x_{1}, x_{2}, x^{0}\right)=0$ for $\left|x_{1}\right|>\varepsilon$ or $\left|x_{2}\right|>\varepsilon$, by (31) we get the representation

$$
\mu_{s}^{1}\left(y_{1}, y^{0}\right)=\nu_{N_{1} N_{2}}\left(y_{1}, y^{0}\right)+\tilde{r}_{N_{1} N_{2}}\left(y_{1}, y^{0}\right)
$$

where

$$
\begin{aligned}
& \nu_{N_{1} N_{2}}\left(y_{1}, y^{0}\right)=\sum_{\substack{\left(z_{1}, q_{1}\right) \in E_{1} \\
z_{1} \leqslant N_{1}}} \sum_{\substack{\left(z_{2}, q_{2}\right) \in E_{2} \\
z_{2} \leqslant N_{2}}} \int_{\mathbb{R}^{n-m-1}}\left(\int_{\substack{\frac{1}{\gamma_{12}} \\
\varepsilon^{-\frac{\gamma_{11}}{\gamma_{12}}}}}^{\varepsilon} b_{z_{1}, q_{1}, z_{2}, q_{2}}\left(y^{0}, x_{m}^{0}, \ldots, x_{n-2}^{0}\right)\right. \\
& \left.t^{z_{2}-\frac{z_{1} \gamma_{12}}{\gamma_{11}}} y_{1}^{\frac{z_{1}}{\gamma_{11}}} \ln ^{q_{2}}|t|\left(\gamma_{11} \ln \left|y_{1}\right|-\frac{\gamma_{12}}{\gamma_{11}} \ln |t|\right)^{q_{1}} \frac{d t}{t}\right) d x_{m}^{0} \ldots d x_{n-2}^{0}
\end{aligned}
$$

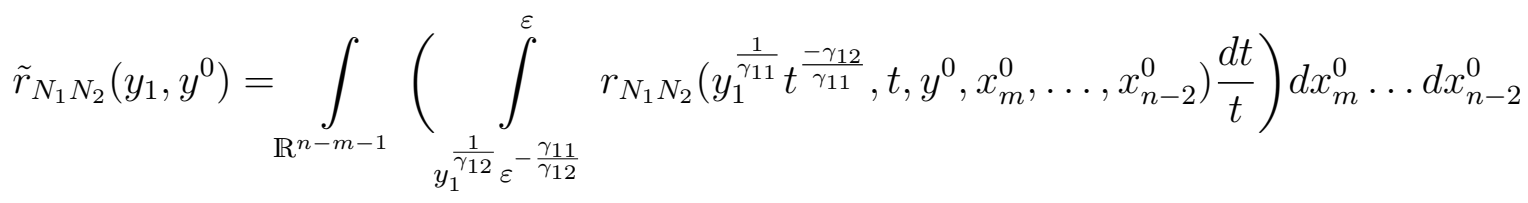


Calculating explicitly the integral over $t$, one can show that function $\nu_{N_{1} N_{2}}\left(y_{1}, y^{0}\right)$ is written as

$$
\begin{aligned}
\nu_{N_{1} N_{2}}\left(y_{1}, y^{0}\right)= & \sum_{\substack{\left(z_{1}, q_{1}\right) \in E_{1} \\
z_{1} \leqslant N_{1}}} d_{z_{1}, q_{1}}^{1}\left(y^{0}\right) y_{1}^{\frac{z_{1}}{\gamma_{11}}} \ln ^{q_{1}}\left|y_{1}\right|+\sum_{\substack{\left(z_{2}, q_{2}\right) \in E_{2} \\
z_{2} \leqslant N_{2}}} d_{z_{2}, q_{2}}^{2}\left(y^{0}\right) y_{1}^{\frac{z_{2}}{\gamma_{12}}} \ln ^{q_{2}}\left|y_{1}\right| \\
& +\sum d_{z_{1}, q_{1}, z_{2}, q_{2}}^{3}\left(y^{0}\right) y_{1}^{\frac{z_{2}}{\gamma_{12}}} \ln ^{q_{1}+q_{2}+1}\left|y_{1}\right|,
\end{aligned}
$$

where the third sum is taken over all collections $\left(z_{1}, q_{1}\right) \in E_{1}, z_{1} \leqslant N_{1},\left(z_{2}, q_{2}\right) \in E_{2}, z_{2} \leqslant N_{2}$ such that $\frac{z_{1}}{\gamma_{11}}=\frac{z_{2}}{\gamma_{12}}$.

Let us estimate $\tilde{r}_{N_{1} N_{2}}$. Splitting the integral over $t$ into a sum of two integrals, we obtain

$$
\tilde{r}_{N_{1} N_{2}}\left(y_{1}, y^{0}\right)=\tilde{r}_{N_{1} N_{2}}^{1}\left(y_{1}, y^{0}\right)+\tilde{r}_{N_{1} N_{2}}^{2}\left(y_{1}, y^{0}\right) \text {, }
$$

where

$$
\begin{aligned}
& \tilde{r}_{N_{1} N_{2}}\left(y_{1}, y^{0}\right)=\int_{\mathbb{R}^{n-m-1}}\left(\int_{y_{1}^{\frac{1}{\gamma_{12}}} \varepsilon^{-\frac{\gamma_{11}}{\gamma_{12}}}}^{y_{1}^{\frac{1}{\gamma_{11}+\gamma_{12}}}} r_{N_{1} N_{2}}\left(y_{1}^{\frac{1}{\gamma_{11}}} t^{\frac{-\gamma_{12}}{\gamma_{11}}}, t, y^{0}, x_{m}^{0}, \ldots, x_{n-2}^{0}\right) \frac{d t}{t}\right) d x_{m}^{0} \ldots d x_{n-2}^{0} \\
& \tilde{r}_{N_{1} N_{2}}^{2}\left(y_{1}, y^{0}\right)=\int_{\mathbb{R}^{n-m-1}}\left(\int_{y_{1}^{\gamma_{11}+\gamma_{12}}}^{\varepsilon} r_{N_{1} N_{2}}\left(y_{1}^{\frac{1}{\gamma_{11}}} t^{\frac{-\gamma_{12}}{\gamma_{11}}}, t, y^{0}, x_{m}^{0}, \ldots, x_{n-2}^{0}\right) \frac{d t}{t}\right) d x_{m}^{0} \ldots d x_{n-2}^{0} .
\end{aligned}
$$

Using (32), we get

$$
\left|\tilde{r}_{N_{1} N_{2}}^{1}\left(y_{1}, y^{0}\right)\right|<C\left(\left|y_{1}\right|^{\frac{\tilde{M}_{1}+N_{2}+1}{\gamma_{11}+\gamma_{12}}}+\left|y_{1}\right|^{\frac{N_{2}+1}{\gamma_{12}}}\right)
$$

To estimate $\tilde{r}_{N_{1} N_{2}}^{2}$, we make use of the similar representation

$$
r_{N_{1} N_{2}}\left(x_{1}, x_{2}, x^{0}\right)=\tilde{r}_{N_{2}}\left(x_{1}, x_{2}, x^{0}\right)+\sum_{\substack{\left(z_{2}, q_{2}\right) \in E_{2} \\ z_{2} \leqslant N_{2}}} \tilde{r}_{z_{2} q_{2} N_{1}}\left(x_{1}, x^{0}\right) x_{2}^{z_{2}} \ln ^{q_{2}}\left|x_{2}\right|,
$$

which implies that there exists $\tilde{M}_{2}$ such that for each $\alpha_{1}, \alpha_{2} \in \mathbb{Z}_{+}$and for each multi-index $\beta_{2}$ there exists a constant $C_{1}$ such that

$$
\left|\left(x_{1} \partial_{x_{1}}\right)^{\alpha_{1}}\left(x_{2} \partial_{x_{2}}\right)^{\alpha_{2}} \partial_{x^{0}}^{\beta_{2}} r_{N_{1} N_{2}}\left(x_{1}, x_{2}, x^{0}\right)\right| \leqslant C_{1}\left|x_{1}\right|^{N_{1}+1}\left|x_{2}\right|^{\tilde{M}_{2}} .
$$

Employing (33), we get

$$
\left|\tilde{r}_{N_{1} N_{2}}^{2}\left(y_{1}, y^{0}\right)\right|<C\left(\left|y_{1}\right|^{\frac{\tilde{M}_{2}+N_{1}+1}{\gamma_{11}+\gamma_{12}}}+\left|y_{1}\right|^{\frac{N_{1}+1}{\gamma_{12}}}\right) .
$$

Thus, we have

$$
\left|\tilde{r}_{N_{1} N_{2}}\left(y_{1}, y^{0}\right)\right|<C\left(\left|y_{1}\right|^{\frac{\tilde{M}_{1}+N_{2}+1}{\gamma_{11}+\gamma_{12}}}+\left|y_{1}\right|^{\frac{N_{2}+1}{\gamma_{12}}}+\left|y_{1}\right|^{\frac{\tilde{M}_{2}+N_{1}+1}{\gamma_{11}+\gamma_{12}}}+\left|y_{1}\right|^{\frac{N_{1}+1}{\gamma_{12}}}\right) .
$$

It implies easily that function $\mu_{s}^{1}\left(y_{1}, y^{0}\right)$ is conormal at $y_{1}=0$ with respect to the index set

$$
E_{1}^{0}=\left\{\left(\frac{z}{\gamma_{11}}, q\right):(z, q) \in E_{1}\right\} \bar{\bigcup}\left\{\left(\frac{z}{\gamma_{12}}, q\right):(z, q) \in E_{2}\right\} .
$$

The case $k_{1}=2, \ell>k$. First, we assume that $k_{1}=2, \ell=3$. In this case, function $\mu_{s}^{1}$ reads as

$$
\mu_{s}^{1}\left(y_{1}, t_{3}, y^{0}\right)=\int_{\mathbb{R} \times \mathbb{R}^{n-m-2}} \mu_{s}\left(y_{1}^{\frac{1}{\gamma_{11}}} t_{2}^{\frac{-\gamma_{12}}{\gamma_{11}}}, t_{2}, t_{3}, y^{0}, x_{m}^{0}, \ldots, x_{n-3}^{0}\right) \frac{d t_{2}}{t_{2}} d x_{m}^{0} \ldots d x_{n-3}^{0} .
$$


Since function $\mu_{s}\left(x_{1}, x_{2}, x_{3}, x^{0}\right)$ is conormal at $\left(x_{1}, x_{2}, x_{3}\right)=(0,0,0)$ with respect to the index family $\left(E_{1}, E_{2}, E_{3}\right)$, we have

$$
\mu_{s}\left(x_{1}, x_{2}, x_{3}, x^{0}\right) \sim \sum_{\left(z_{3}, q_{3}\right) \in E_{3}} a_{z_{3}, q_{3}}\left(x_{1}, x_{2}, x^{0}\right) x_{3}^{z_{3}} \ln ^{q_{3}}\left|x_{3}\right|,
$$

where $a_{z_{3}, q_{3}}\left(x_{1}, x_{2}, x^{0}\right)$ are conormal functions at $\left(x_{1}, x_{2}\right)=(0,0)$ with respect to the index family $\left(E_{1}, E_{2}\right)$. By definition, for each natural $N$ the representation

$$
\mu_{s}\left(x_{1}, x_{2}, x_{3}, x^{0}\right)=\sum_{\substack{\left(z_{3}, q_{3}\right) \in E_{3} \\ z_{3} \leqslant N}} a_{z_{3}, q_{3}}\left(x_{1}, x_{2}, x^{0}\right) x_{3}^{z_{3}} \ln ^{q_{3}}\left|x_{3}\right|+r_{N}\left(x_{1}, x_{2}, x_{3}, x^{0}\right)
$$

holds true. In accordance with (34), $\mu_{s}^{1}$ is represented as $\mu_{s}^{1}=\nu_{N}+\tilde{r}_{N}$, where

$$
\nu_{N}\left(y_{1}, t_{3}, y^{0}\right)=\sum_{\substack{\left(z_{3}, q_{3}\right) \in E_{3} \\ z_{3} \leqslant N}} b_{z_{3}, q_{3}}\left(y_{1}, y^{0}\right) t_{3}^{z_{3}} \ln ^{q_{3}}\left|t_{3}\right|
$$

where

$$
b_{z_{3}, q_{3}}\left(y_{1}, y^{0}\right)=\int_{\mathbb{R} \times \mathbb{R}^{n-m-2}} a_{z_{3}, q_{3}}\left(y_{1}^{\frac{1}{\gamma_{11}}} t_{2}^{\frac{-\gamma_{12}}{\gamma_{11}}}, t_{2}, y^{0}, x_{m}^{0}, \ldots, x_{n-3}^{0}\right) \frac{d t_{2}}{t_{2}} d x_{m}^{0} \ldots d x_{n-3}^{0} .
$$

and

$$
\tilde{r}_{N}\left(y_{1}, t_{3}, y^{0}\right)=\int_{\mathbb{R} \times \mathbb{R}^{n-m-2}} r_{N}\left(y_{1}^{\frac{1}{\gamma_{11}}} t_{2}^{\frac{-\gamma_{12}}{\gamma_{11}}}, t_{2}, t_{3}, y^{0}, x_{m}^{0}, \ldots, x_{n-3}^{0}\right) \frac{d t_{2}}{t_{2}} d x_{m}^{0} \ldots d x_{n-3}^{0} .
$$

By Proposition 2 in the case $k_{1}=\ell=2$, functions $b_{z_{3}, q_{3}}\left(y_{1}, y^{0}\right)$ are conormal at $y_{1}=0$ with respect to index set $E_{1}^{0}$. Therefore, function $\nu_{N}\left(y_{1}, t_{3}, y^{0}\right)$ is conormal at $\left(y_{1}, t_{3}\right)=(0,0)$ with respect to the index set $\left(E_{1}^{0}, E_{3}\right)$.

By definition, there exist $M_{1}$ and $M_{2}$ such that, for each $\alpha_{1}, \alpha_{2}, \alpha_{3} \in \mathbb{Z}_{+}$and for each multiindex $\beta_{2}$, there exists a constant $C_{1}$ such that

$$
\left|\left(x_{1} \partial_{x_{1}}\right)^{\alpha_{1}}\left(x_{2} \partial_{x_{2}}\right)^{\alpha_{2}}\left(x_{3} \partial_{x_{3}}\right)^{\alpha_{3}} \partial_{x^{0}}^{\beta_{2}} r_{N}\left(x_{1}, x_{2}, x_{3}, x^{0}\right)\right|<C_{1}\left|x_{1}\right|^{M_{1}}\left|x_{2}\right|^{M_{2}}\left|x_{3}\right|^{N+1} .
$$

Using these estimates, one can show that there exists a constant $M$ such that for each $\alpha_{1}, \alpha_{2} \in$ $\mathbb{Z}_{+}$and for each multi-index $\beta$, there exists a constant $C_{1}$ such that

$$
\left|\left(y_{1} \partial_{y_{1}}\right)^{\alpha_{1}}\left(t_{3} \partial_{t_{3}}\right)^{\alpha_{2}} \partial_{y^{0}}^{\beta} \tilde{r}_{N}\left(y_{1}, t_{3}, y^{0}\right)\right|<C_{1}\left|y_{1}\right|^{M}\left|t_{3}\right|^{N+1}
$$

It completes the proof of Proposition 2 in the case $k_{1}=2, \ell=3$.

The case $k_{1}=2$ and arbitrary $\ell>k$ is proved in the same way by induction in $\ell$.

The proof of Proposition 2 for arbitrary $k_{1}$ and $\ell \geqslant k_{1}$ is completed by induction in $k_{1}$.

Suppose that Proposition 2 is valid for each $k_{1}<k$, for each $\ell \geqslant k_{1}$, and for each function $\mu_{s}$. Let us prove Proposition 2 for $k_{1}=k$, for each $\ell \geqslant k_{1}$, and for each function $\mu_{s}$.

To begin with, we consider the case $k_{1}=\ell=k$. In this case, we represent function $\mu_{s}^{1}$ given by (29) as follows:

$$
\mu_{s}^{1}\left(y_{1}, y^{0}\right)=\int_{\mathbb{R}} \widetilde{\mu}\left(y_{1} t_{k}^{-\gamma_{1 k}}, t_{k}, y^{0}\right) \frac{d t_{k}}{t_{k}}
$$

where

$$
\begin{array}{r}
\widetilde{\mu}\left(z_{1}, t_{k}, y^{0}\right)=\int_{\mathbb{R}^{k-2} \times \mathbb{R}^{n-m-k+1}} \mu_{s}\left(z_{1}^{\frac{1}{\gamma_{11}}} t_{2}^{-\frac{\gamma_{12}}{\gamma_{11}}} \ldots t_{k-1}^{-\frac{\gamma_{1, k-1}}{\gamma_{11}}}, t_{2}, \ldots, t_{k}, y^{0}, x_{m}^{0}, \ldots, x_{n-k}^{0}\right) \\
\frac{d t_{2}}{t_{2}} \ldots \frac{d t_{k-1}}{t_{k-1}} d x_{m}^{0} \ldots d x_{n-k}^{0} .
\end{array}
$$


Proposition 2 in the case $k_{1}=k-1, \ell=k$ implies that function $\widetilde{\mu}\left(z_{1}, t_{k}\right)$ is conormal in $\left(z_{1}, t_{k}\right)$ with respect to the index family $\left(\widetilde{E}_{1}^{0}, E_{k}\right)$, where

$$
\widetilde{E}_{1}^{0}=\bar{\bigcup}_{j=1, \ldots, k-1}\left\{\left(\frac{z}{\gamma_{1 j}}, q\right):(z, q) \in E_{j}\right\} .
$$

Applying Proposition 2 in the case $k_{1}=\ell=2$, we obtain that function $\nu_{s}\left(y_{1}, y^{0}\right)$ is conormal in $y_{1}$ with respect to the index set

$$
\widetilde{E}_{1}^{0} \bar{\bigcup}\left\{\left(\frac{z}{\gamma_{1 k}}, q\right):(z, q) \in E_{k}\right\}=E_{1}^{0} .
$$

The case $k_{1}=k$ and arbitrary $\ell>k_{1}$ is proved as above by induction in $\ell$. The proof of Proposition 2 is completed.

Proof of Theorem 6 in the case $\ell_{0}=2$. We choose an adapted at $p_{0}$ coordinate system with coordinates $\left(y_{1}, y_{2}, y^{0}\right) \in D_{1}^{0} \times D_{2}^{0} \subset \mathbb{R}^{2} \times \mathbb{R}^{m-2}$ and $p \in X$ such that $f(p)=p_{0}$. We suppose that $p \in X_{1} \cap \ldots \cap X_{\ell}$ and $p \notin X_{\ell+1} \cup \ldots \cup X_{r}$. We choose an adapted at $p$ coordinate system with coordinates $\left(x, x^{0}\right) \in D_{1} \times D_{2} \subset \mathbb{R}^{\ell} \times \mathbb{R}^{n-\ell}$. By assumption, without loss of generality we can assume that in these coordinate systems map $f$ is written as $\left(y_{1}, y_{2}, y^{0}\right)=f\left(x, x^{0}\right)$, where $y_{1}=b_{1}\left(x, x^{0}\right) x_{1}^{\gamma_{11}} \ldots x_{k_{1}}^{\gamma_{1 k_{1}}}, y_{2}=b_{2}\left(x, x^{0}\right) x_{k_{1}+1}^{\gamma_{2, k_{1}+1}} \ldots x_{k_{2}}^{\gamma_{2 k_{2}}}$; functions $b_{1}$ and $b_{2}$ are smooth and non-vanishing; $\gamma_{11}, \ldots, \gamma_{1 k_{1}}, \gamma_{2, k_{1}+1}, \ldots, \gamma_{2, k_{2}}>0, k_{1}<k_{2} \leqslant \ell ; y^{0}=g\left(x, x^{0}\right)$. As in the case $\ell_{0}=1$, without loss of generality we can assume that $b_{1}\left(x, x^{0}\right) \equiv b_{2}\left(x, x^{0}\right) \equiv 1$.

By Condition (4) of Definition 13, we have rank $\left(\frac{\partial g}{\partial x^{0}}\right)=m-2$. Hence, one can choose an adapted at $p_{0}$ coordinate system such that map $g$ becomes a projection:

$$
g\left(x, x^{0}\right)=\left(x_{1}^{0}, \ldots, x_{m-2}^{0}\right), \quad x \in D_{1}, \quad x^{0} \in D_{2} .
$$

By compactness of $X$, there exists a finite family of neighborhoods $V_{p_{s}}, s=1, \ldots, d$, such that $X=\left(X \backslash f^{-1}\left(p_{0}\right)\right) \bigcup \bigcup_{s=1}^{d} V_{p_{s}}$. Let $\psi_{s} \in C^{\infty}(X), s=0, \ldots, d$ be a smooth partition of unity subordinated to this covering: $\operatorname{supp} \psi_{0} \subset X \backslash f^{-1}\left(p_{0}\right), \operatorname{supp} \psi_{s} \subset V_{p_{s}}$ for $s=1, \ldots, d$, $\psi_{s} \geqslant 0, \sum_{s=0}^{d} \psi_{s}=1$. There exists a neighborhood $U_{p_{0}}$ of $p_{0}$ such that $\sum_{s=1}^{d} \psi_{s}(m)=1$ for each $m \in f^{-1}\left(U_{p_{0}}\right)$.

As above, we assume that bundle $G$ is trivial and $\mu$ is a density on $X$. In coordinate neighborhood $V_{p_{s}}$, density $\mu$ is written as

$$
\mu=\mu\left(x, x^{0}\right)\left|\frac{d x}{x} d x^{0}\right| .
$$

We choose $\varphi \in C_{0}^{\infty}(Y)$ such that $\operatorname{supp} \varphi \subset U_{p_{0}}$. Denoting

we get

$$
\mu_{s}\left(x, x^{0}\right)=\frac{1}{\gamma_{11} \gamma_{2, k_{1}+1}} \psi_{s}\left(x, x^{0}\right) \mu\left(x, x^{0}\right),
$$

$$
\begin{aligned}
\left\langle f_{*} \mu, \varphi\right\rangle=\gamma_{11} \gamma_{2, k_{1}+1} \sum_{s=1}^{d} \int_{\mathbb{R}^{\ell} \times \mathbb{R}^{n-\ell}} & \mu_{s}\left(x, x^{0}\right) \\
& \times \varphi\left(x_{1}^{\gamma_{11}} \ldots x_{k_{1}}^{\gamma_{1 k_{1}}}, x_{k_{1}+1}^{\gamma_{2, k_{1}+1}} \ldots x_{\ell}^{\gamma_{2, k_{2}}}, x_{1}^{0}, \ldots, x_{m-2}^{0}\right) \frac{d x}{x} d x^{0} .
\end{aligned}
$$

Making the change of variables

$$
\begin{gathered}
y_{1}=x_{1}^{\gamma_{11}} \ldots x_{k_{1}}^{\gamma_{1 k_{1}}} ; \quad y_{2}=x_{k_{1}+1}^{\gamma_{2, k_{1}+1}} \ldots x_{k_{2}}^{\gamma_{2 k_{2}}} ; \quad y^{0}=\left(x_{1}^{0}, \ldots, x_{m-2}^{0}\right) \\
t_{j}=x_{j} \quad \forall j=2, \ldots, k_{1}, k_{1}+2, \ldots, \ell
\end{gathered}
$$


in the integral, we obtain

$$
\begin{aligned}
& \left\langle f_{*} \mu, \varphi\right\rangle=\sum_{s=1}^{d} \int_{\mathbb{R}^{\ell} \times \mathbb{R}^{n-\ell}} \mu_{s}\left(y_{1}^{\frac{1}{\gamma_{11}}} t_{2}^{\frac{-\gamma_{12}}{\gamma_{11}}} \ldots t_{k_{1}}^{-\frac{\gamma_{1 k_{1}}}{\gamma_{11}}}, t_{2}, \ldots, t_{k_{1}},\right. \\
& \left.y_{2}^{\frac{1}{\gamma_{2, k_{1}+1}}} t_{k_{1}+2}^{-\frac{\gamma_{2, k_{1}+2}}{\gamma_{2}+k_{1}+1}} \ldots t_{k_{2}}^{-\frac{\gamma_{2 k_{2}}}{\gamma_{2, k_{1}+1}}}, t_{k_{1}+2}, \ldots, t_{\ell}, y^{0}, x_{m}^{0}, \ldots, x_{n-\ell}^{0}\right) \varphi\left(y_{1}, y_{2}, y^{0}\right) \\
& \frac{d y_{1}}{y_{1}} \frac{d y_{2}}{y_{2}} \frac{d t_{2}}{t_{2}} \ldots \frac{d t_{k_{1}}}{t_{k_{1}}} \frac{d t_{k_{1}+2}}{t_{k_{1}+2}} \ldots \frac{d t_{\ell}}{t_{\ell}} d y^{0} d x_{m}^{0} \ldots d x_{n-\ell}^{0} .
\end{aligned}
$$

Hence, for each $\left(y_{1}, y_{2}, y^{0}\right)$, density $f_{*} \mu$ is given by

$$
f_{*} \mu=\sum_{s=1}^{d} \nu_{s}\left(y_{1}, y_{2}, y^{0}\right)\left|\frac{d y_{1}}{y_{1}} \frac{d y_{2}}{y_{2}} d y^{0}\right|,
$$

where functions $\nu_{s}\left(y_{1}, y_{2}, y^{0}\right)$ read as

$$
\begin{aligned}
\nu_{s}\left(y_{1}, y_{2}, y^{0}\right)= & \int_{\mathbb{R}^{\ell-2} \times \mathbb{R}^{n-m-\ell+1}} \mu_{s}\left(y_{1}^{\frac{1}{\gamma_{11}}} t_{2}^{\frac{-\gamma_{12}}{\gamma_{11}}} \ldots t_{k_{1}}^{-\frac{\gamma_{1 k_{1}}}{\gamma_{11}}}, t_{2}, \ldots, t_{k_{1}},\right. \\
& \left.y_{2}^{\frac{1}{\gamma_{2, k_{1}+1}}} t_{k_{1}+2}^{-\frac{\gamma_{2, k_{1}+2}}{\gamma_{2}+1}} \ldots t_{k_{2}}^{-\frac{\gamma_{2 k_{2}}}{\gamma_{2, k_{1}+1}}}, t_{k_{1}+2}, \ldots, t_{\ell}, y^{0}, x_{m}^{0}, \ldots, x_{n-\ell}^{0}\right) \\
& \frac{d t_{2}}{t_{2}} \ldots \frac{d t_{k_{1}}}{t_{k_{1}}} \frac{d t_{k_{1}+2}}{t_{k_{1}+2}} \ldots \frac{d t_{\ell}}{t_{\ell}} d x_{m}^{0} \ldots d x_{n-\ell}^{0} .
\end{aligned}
$$

Since for $j=k_{2}+1, \ldots, \ell$ we have inf $E_{j}>0$, the integral in the last formula converges, therefore, $\nu_{s}\left(y_{1}, y_{2}, y^{0}\right)$ is a smooth function for $y_{1} y_{2} \neq 0$.

Let us prove that function $\nu_{s}\left(y_{1}, y_{2}, y_{0}\right)$ is conormal at $(0,0)$ with respect to the index family $\left(E_{1}^{0}, E_{2}^{0}\right)$. We write the function $\nu_{s}\left(y_{1}, y_{2}, y_{0}\right)$ as

$$
\nu_{s}\left(y_{1}, y_{2}, y^{0}\right)=\int_{\mathbb{R}^{\ell-k_{2}}} \chi_{1}\left(y_{1}, y_{2}, t_{k_{2}+1}, \ldots, t_{\ell}, y^{0}\right) \frac{d t_{k_{2}+1}}{t_{k_{2}+1}} \ldots \frac{d t_{\ell}}{t_{\ell}},
$$

where

$$
\begin{aligned}
\chi_{1}\left(y_{1}, y_{2}, t_{k_{2}+1}, \ldots, t_{\ell}, y^{0}\right) \\
=\int_{\mathbb{R}^{k_{2}-k_{1}-1}} \chi\left(y_{1}, y_{2}^{\frac{1}{\gamma_{2, k_{1}+1}}} t_{k_{1}+2}^{-\frac{\gamma_{2, k_{1}+2}}{\gamma_{2}+1}} \ldots t_{\ell}^{-\frac{\gamma_{2 \ell}}{\gamma_{2, k_{1}+1}}}, t_{k_{1}+2}, \ldots, t_{\ell}, y^{0}\right) \frac{d t_{k_{1}+2}}{t_{k_{1}+2}} \ldots \frac{d t_{k_{2}}}{t_{k_{2}}},
\end{aligned}
$$

and

$$
\begin{aligned}
& \chi\left(y_{1}, \tau_{k_{1}+1}, \ldots, \tau_{\ell}, y^{0}\right)= \int_{\mathbb{R}^{k_{1}-1} \times \mathbb{R}^{n-m-\ell+1}} \mu_{s}\left(y_{1}^{\frac{1}{\gamma_{11}}} t_{2}^{\frac{-\gamma_{12}}{\gamma_{11}}} \ldots t_{k_{1}}^{-\frac{\gamma_{1 k_{1}}}{\gamma_{11}}}, t_{2}, \ldots, t_{k_{1}},\right. \\
&\left.\tau_{k_{1}+1}, \ldots, \tau_{\ell}, y^{0}, x_{m}^{0}, \ldots, x_{n-\ell}^{0}\right) \frac{d t_{2}}{t_{2}} \ldots \frac{d t_{k_{1}}}{t_{k_{1}}} d x_{m}^{0} \ldots d x_{n-\ell}^{0} .
\end{aligned}
$$

It follows from Proposition 2 that $\chi\left(y_{1}, \tau_{k_{1}+1}, \tau_{k_{1}+2}, \ldots, \tau_{\ell}, y^{0}\right)$ is conormal in the variables $\left(y_{1}, \tau_{k_{1}+1}, \ldots, \tau_{\ell}\right)$ with respect to the index family $\left(E_{1}^{0}, E_{k_{1}+1}, \ldots, E_{\ell}\right)$, and $\chi_{1}\left(y_{1}, y_{2}, t_{k_{2}+1}, \ldots, t_{\ell}, y^{0}\right)$ is conormal in the variables $\left(y_{1}, y_{2}, t_{k_{2}+1}, \ldots, t_{\ell}\right)$ with respect to the index family $\left(E_{1}^{0}, E_{2}^{0}, E_{k_{2}+1}, \ldots, E_{\ell}\right)$. The conormality of function $\nu_{s}\left(y_{1}, y_{2}, y^{0}\right)$ at $\left(y_{1}, y_{2}\right)=$ $(0,0)$ with respect to the index family $\left(E_{1}^{0}, E_{2}^{0}\right)$ follows from Theorem 6 in the case $\ell_{0}=0$ in view of the condition $\inf E_{j}>0, \forall j=k_{2}+1, \ldots, \ell$. Thus, the case $\ell_{0}=2$ is proved.

The proof of Theorem 6 in the case of arbitrary $\ell_{0}>2$ is given in the same way by induction in $\ell_{0}$. 


\section{BIBLIOGRAPHY}

1. J. Álvarez López, Yu.A. Kordyukov. Distributional Betti numbers of transitive foliations of codimension one. In: Foliations: Geometry and Dynamics, World Sci. Publishing, River Edge, NJ, 159-183 (2002).

2. S. Soboleff. Sur un problème limite pour les équations polyharmoniques // Rec. Math. [Mat. Sbornik] N.S., 2(44):3, 465-499 (1937). (in Russian).

3. B.Yu. Sternin. Elliptic and parabolic problems on manifolds with a boundary consisting of components of different dimension // Trudy Moskov. Mat. Obsh. 15 346-382 (1966). (in Russian).

4. B.Yu. Sternin, V.E. Shatalov. Relative elliptic theory and the Sobolev problem // Sb. Math., 187:11, 1691-1720 (1996).

5. B.Yu. Sternin. A relative elliptic theory, and S.L. Sobolev's problem // Sov. Math., Dokl. 17, 13061309 (1976).

6. B.Yu. Sternin. S.L. Sobolev type problems in the case of submanifolds with multidimensional singularities // Soviet Math. Dokl. 10, 1499-1502 (1970).

7. B.Yu. Sternin. Elliptic morphisms (riggings of elliptic operators) for submanifolds with singularities // Soviet Math. Dokl. 12, 1338-1343 (1971).

8. B.Yu. Sternin. Elliptic theory on compact manifolds with singularities. Moskov. Inst. Elektron. Mashinostroen., Moscow (1974). (in Russian).

9. A.Yu. Savin, B.Yu. Sternin. Elliptic translators on manifolds with multidimensional singularities // Differ. Equats. 49:4, 494-509 (2013).

10. A.Yu. Savin, B.Yu. Sternin. Index of Sobolev problems on manifolds with many-dimensional singularities // Differ. Equats. 50:2, 232-245 (2014).

11. R.B. Melrose. Pseudodifferential operators, corners and singular limits // Proceedings of the International Congress of Mathematicians, Vol. I, II. Math. Soc. Japan, Tokyo. 217-234 (1991).

12. R.B. Melrose. Calculus of conormal distributions on manifolds with corners // Inter. Math. Res. Notices. 1992:3, 51-61 (1992).

13. R.B. Melrose. The Atiyah-Patodi-Singer index theorem. Research Notes in Mathematics, V. 4. A.K. Peters, Ltd., Wellesley, MA (1993).

14. V.E. Nazaikinskii, A.Yu. Savin, B.Yu. Sternin. Noncommutative geometry and classification of elliptic operators // J. Math. Sci. 164:4, 603-636 (2010).

15. D. Grieser. Basics of the b-calculus. In: "Approaches to singular analysis", Oper. Theory Adv. Appl., V. 125, Birkhauser, Basel. 30-84 (2001).

16. V.E. Nazaikinskii, A.Yu. Savin, B.W. Schulze, B.Yu. Sternin. Elliptic theory on singular manifolds. Chapman Hall//CRC, Boca Raton, FL (2006).

Yuri Arkad'evich Kordyukov,

Institute of mathematics, Russian Academy of Sciences,

112, Chernyshevsky str.,

450008 Ufa, Russia

E-mail: yurikor@matem.anrb.ru

Victor Alexandrovich Pavlenko,

Bashkir State Agrarian University,

34 50-letiya Oktyabrya Str.,

450001 Ufa, Russia

E-mail: PVA100186@mail.ru 